\title{
Bounds for capacities in terms of asymmetry
}

Tilak Bhattacharya and Allen Weitsman

\section{Introduction.}

In [6], a study was initiated by R. Hall, W. Hayman, and A. Weitsman relating the asymmetry of a set to various set parameters such as the diameter, isoperimetric constant, and capacity. For a compact set $\Omega$ in $\mathbb{R}^{n}$, let $V(\Omega)$ denote the volume of $\Omega$, and $B(x, \rho)$ the ball of radius $\rho$ centered at $x$ and volume $V(\Omega)$. The asymmetry $\alpha=\alpha(\Omega)$ is then defined by

$$
\alpha=\inf _{x} \frac{V(\Omega \backslash B(x, \rho))}{V(\Omega)}, \quad \rho=\sqrt[n]{\frac{V(\Omega)}{V(B(0,1))}} .
$$

In $\mathbb{R}^{2}$, we shall use $A(\Omega)$ to denote the area of $\Omega$. It is clear that $\alpha=0$ when $\Omega$ is a ball.

Let $\operatorname{Cap}(\Omega)$ denote the logarithmic capacity of a set $\Omega$ in $\mathbb{R}^{2}$. In [6] it was shown that there exists an absolute constant $K_{0}$ such that

$$
\operatorname{Cap}(\Omega) \geq\left(1+K_{0} \alpha(\Omega)^{3}\right) \sqrt{\frac{A(\Omega)}{\pi}} .
$$

This was improved by W. Hansen and N. Nadirashvili in [7] where it was shown that there exists an absolute constant $K_{1}$ such that

$$
\operatorname{Cap}(\Omega) \geq\left(1+K_{1} \alpha(\Omega)^{2}\right) \sqrt{\frac{A(\Omega)}{\pi}} .
$$


The inequality (1.3) was conjectured by L. E. Fraenkel and, as noted in [6], the exponent 2 in (1.3) is sharp. The proof in [7] relies on an inequality between capacity and moment of inertia which had been proved by Pólya and Szegö [10, p. 126] for connected sets. For general sets, this inequality had remained open until Hansen and Nadirashvili's ingenious proof in [7]. They also showed that, in (1.3), $K_{1} \geq 1 / 4$. The proofs in [6] are based on estimates for condensers.

In this work we shall prove an analogue of (1.3) for $p$-capacities of condensers in the plane. The $p$-capacities have been studied extensively in recent years, especially in connection with degenerate nonlinear elliptic partial differential equations [10]. Since such capacities are very hard to compute exactly ( $c f .[10$, p. 35$]$ ), we shall develop a perturbative method to obtain approximations in terms of asymmetry.

A condenser $\Gamma=\Gamma\left(\Omega, \Omega^{\prime}\right)$ in $\mathbb{R}^{2}$ consists of a compact set $\Omega$ and a disjoint closed unbounded set $\Omega^{\prime}$. The $p$-capacity $(1<p<\infty)$ of the condenser is then

$$
\operatorname{Cap}_{p}(\Gamma)=\inf \iint_{\mathbb{R}^{2}}|D u|^{p} d x d y,
$$

the infimum being taken over all functions $u$ absolutely continuous in $\mathbb{R}^{2}$, with $u=0$ on $\Omega$ and $u=1$ on $\Omega^{\prime}$. When $p=2$, the minimizer is the harmonic function in $\mathbb{R}^{2} \backslash\left(\Omega \cup \Omega^{\prime}\right)$ having the prescribed boundary values. For other values of $p$, the minimizer satisfies the " $p$-Laplace equation", namely, $\operatorname{div}\left(|D u|^{p-2} D u\right)=0$. Although solutions to this equation have only locally Hölder continuous first derivatives [12], they do retain a maximum principle, and the critical values are discrete in $\mathbb{R}^{2} \backslash\left(\Omega \cup \Omega^{\prime}\right)$ [13]. Furthermore, $u$ is analytic near points where $D u \neq 0$ (cf. [11, p. 208]). We will consider $p$-capacities of condensers $\Gamma=$ $\Gamma\left(\Omega, \Omega^{\prime}\right)$ where $A(\Omega)=1$ and $A\left(\mathbb{R}^{2} \backslash \Omega^{\prime}\right)=4$. The main result of this work is

Theorem 1. Let $1<p<\infty$. There exist constants $K_{p}$ depending only on $p$, such that

$$
\operatorname{Cap}_{p}(\Gamma) \geq\left(1+K_{p} \alpha(\Omega)^{2}\right) \operatorname{Cap}_{p}\left(\Gamma^{*}\right),
$$

where $\Gamma$ is as above, and $\Gamma^{*}=\Gamma\left(B(0,1 / \sqrt{\pi}), \mathbb{R}^{2} \backslash B(0,2 / \sqrt{\pi})\right)$.

The $p$-capacity of $\Gamma^{*}$ is given explicitly by

$$
\operatorname{Cap}_{p}\left(\Gamma^{*}\right)=\left(\int_{1}^{4} \phi(t) d t\right)^{1-p}
$$


where $\phi(t)=\phi_{p}(t)=(4 \pi t)^{p / 2(1-p)}$.

In Section 9 we show that the exponent 2 in (1.5) is sharp.

The methods of this paper can be extended to cover condensers whose inner and outer boundaries exhibit asymmetries, but at a cost of much routine and tedious work. Also, (1.5) in case $p=2$ can be used to give (1.3). In Section 10 we outline this proof. Although it is impossible, due to the intricacies of the proof, to give any meaningful numerical bounds on the constants $K_{p}$ in (1.5), with additional work one could allow $\Omega$ and $\Omega^{\prime}$ to vary in size. The influence on the constants $K_{p}$ will be discussed in Section 11.

In higher dimensions only partial results have been obtained relating capacities to asymmetry. Under the assumption of convexity on $\Omega$, if $\operatorname{Cap}(\Omega)$ denotes the Newtonian capacity of $\Omega$, then in [6] the inequality corresponding to (1.3) with exponent $n+1$ on $\alpha$ was obtained. This was improved by Hansen and Nadirashvili [7], [8], again for convex sets, also replacing the asymmetry by the quantity

$$
d_{e}(\Omega)=\frac{R_{0}(\Omega)}{R(\Omega)}-1
$$

where $R_{0}$ is the outradius of $\Omega$ and $R(\Omega)$ is the radius of the ball having volume $V(\Omega)$. They proved that for small $d=d_{e}(\Omega)$,

$$
\frac{\operatorname{Cap}(\Omega)}{\operatorname{Cap}(B(0, \rho))} \geq \begin{cases}1+A \frac{d^{3}}{\log 1 / d}, & n=3, \\ 1+A_{n} d^{(n+3) / 2}, & n \geq 4,\end{cases}
$$

where $V(B(0, \rho))=V(\Omega)$.

The main challenge which lies ahead is to determine the effect of asymmetry on Newtonian capacity without the assumption of convexity. Although $\alpha<d_{e}$, and (1.7) is close to best possible for convex sets [8, p. 8], the quantity $d_{e}$ has no relevance in the study of general $\Omega$. This stems from the fact that line segments have capacity 0 in $\mathbb{R}^{n}$ for $n \geq 3$, and so $d_{e}$ can be depressed with negligible effect on the capacity. On the other hand, the notion of asymmetry, which seems to have been introduced in this context by Fraenkel, remains a natural measure of distortion. It seems reasonable to us to conjecture that

$$
\frac{\operatorname{Cap}(\Omega)}{\operatorname{Cap}(B(0, \rho))} \geq\left(1+D_{n} \alpha^{2}\right)
$$

for constants $D_{n}$ where again $V(B(0 ; \rho))=V(\Omega)$. 
In an unpublished work, Fraenkel has verified (1.8) for starlike regions close to a ball in $\mathbb{R}^{3}$. However, contrary to the remark attributed to the second author in [9], no general bounds on Newtonian capacity in terms of asymmetry appear to be known. It would be interesting to obtain an inequality of the type (1.8) with some exponent on $\alpha$, but with no assumption of convexity on $\Omega$.

There are two natural avenues of approach to this problem. The first would be to prove an inequality for the moment of inertia $I(\Omega)$ of $\Omega$ about its centroid in terms of $\operatorname{Cap}(\Omega)$ as was done in $\mathbb{R}^{2}$ by Hansen and Nadirashvili. If one could prove the hypothetical inequality

$$
\operatorname{Cap}(\Omega)^{n+2} \geq \frac{(n+2)}{\sigma_{n}} I(\Omega)
$$

where $\sigma_{n}$ is the $(n-1)$-Hausdorff measure of the unit sphere, and where we have normalized so that the capacity of a ball is its radius, then (1.8) would follow easily from

$$
I(\Omega) \geq I(B)\left[1+\frac{n+2}{n^{2}} \alpha^{2}\right],
$$

where $B$ is the ball of volume $V(\Omega)$. Inequality (1.9) is a natural analogue of the inequality of Hansen and Nadirashvili in $\mathbb{R}^{n}$.

Another possible approach is along the lines of the present paper, especially in view of the recent results of Hall [5] which give the influence of the asymmetry on the usual isoperimetric inequality. With this in mind, the results of this paper, in particular the symmetrization method introduced in Section 3 can be adapted to $\mathbb{R}^{n}$ for $n \geq 3$ as long as $p=2$. The difficulty arises in Section 6 where one needs to prove that if the asymmetry is very small, most of $\Omega$ is a set whose boundary lies between two very close concentric balls. The present argument relies on the Bonnesen type inequalities (2.2)-(2.4), and it seems difficult to extend this type of argument to higher dimensions.

In the case of $p$-capacities of condensers in $\mathbb{R}^{n}, n>2$, nothing seems to be known regarding an analogue of (1.5), even under the additional assumption of convexity. The problem is more difficult especially because there are no known bounds on the sets of critical points, and in particular whether or not such sets are of measure zero. Nevertheless, it seems likely that (1.5) will continue to hold. More precisely, let $R_{n}$ be such that $V\left(B\left(0, R_{n}\right)\right)=1, \Gamma=\Gamma\left(\Omega, \Omega^{\prime}\right)$ be a condenser with $V(\Omega)=1$, and $V\left(\mathbb{R}^{n} \backslash \Omega^{\prime}\right)=2^{n}$. Let $\Gamma^{*}$ denote the condenser 
$\Gamma\left(\bar{B}\left(0, R_{n}\right), \mathbb{R}^{n} \backslash B\left(0,2 R_{n}\right)\right)$. Then we conjecture that there is a $K_{p}>0$, depending only on $p$, such that

$$
\operatorname{Cap}_{p}(\Gamma) \geq\left(1+K_{p} \alpha^{2}\right) \operatorname{Cap}_{p}\left(\Gamma^{*}\right) .
$$

We have divided our work as follows. In Section 2, we state and prove some preliminary results required in the proof of Theorem 1 . We also discuss our strategy for achieving the proof of Theorem 1. In Section 3, we introduce a new symmetrization technique. Based on this, we prove a perturbation lemma for 2-capacity in Section 4. The proof of Theorem 1 involves considering several independent cases and is spread over sections 5-8. In Section 9, we present an example to prove the sharpness of the exponent 2 in (1.5); Section 10 contains a proof of (1.3) based on the techniques developed in this paper. Finally, in Section 11, we indicate how our result in (1.5) is modified when the ratio of the areas of the sets involved is different from 4 .

As in [6], our proofs will rely in part on connections with the isoperimetric inequality. These ideas have been useful in a number of studies ( $c f .[3],[4],[14],[17])$.

\section{Preliminary results.}

We may assume that the sets we are working with are bounded by a finite number of rectifiable curves. Let $D$ be such a set and $L(\partial D)$ denote the length of its boundary. Then it is proved in [6, Lemma 2.1] that

$$
L(\partial D)^{2} \geq 4 \pi\left(1+\frac{\alpha(D)^{2}}{6}\right) A(D)
$$

In proving (2.1), use was made of relations between the inradius $R_{i}$ and outradius $R_{o}$ of $D$. Results of this type are collected in [15]. In this paper, we shall have occasion to use the fact $[15$, p. 3-4] that if $D$ is bounded by a rectifiable Jordan curve, then

$$
\begin{gathered}
L(\partial D)^{2}-4 \pi A(D) \geq \pi^{2}\left(R_{o}-R_{i}\right)^{2}, \\
R_{o} \leq \frac{1}{2 \pi}\left(L(\partial D)+\sqrt{L(\partial D)^{2}-4 \pi A(D)}\right),
\end{gathered}
$$


and

$$
R_{i} \geq \frac{1}{2 \pi}\left(L(\partial D)-\sqrt{L(\partial D)^{2}-4 \pi A(D)}\right)
$$

Proposition 2.1. Suppose that $D$ is a bounded open set and $D=$ $\cup_{i=1}^{\infty} D_{i}$, where the $D_{i}$ 's are pairwise disjoint components of D, labelled such that $A\left(D_{1}\right) \geq A\left(D_{2}\right) \geq \cdots$. If $0<\delta<1 / 4$, and

$$
A\left(D_{1}\right) \leq(1-\delta) A(D)
$$

then

$$
L(\partial D)^{2} \geq 4 \pi(1+\sqrt{\delta}) A(D)
$$

Proof. We assume that the perimeter of each $D_{i}$ is finite. Set $x_{i}=$ $A\left(D_{i}\right), i=1,2, \ldots$, so that $\sum_{i=1}^{\infty} x_{i}=A(D)$, and $x_{1} \geq x_{2} \geq x_{3} \geq \cdots$. Also

$$
x_{1} \leq(1-\delta) A(D)
$$

We first consider the case when $x_{1} \geq \delta A(D)$. Employing the isoperimetric inequality, we have

$$
\begin{aligned}
L(\partial D)^{2} & =\left(L\left(\partial D_{1}\right)+\sum_{i=2}^{\infty} L\left(\partial D_{i}\right)\right)^{2} \\
& \geq L\left(\partial D_{1}\right)^{2}+\sum_{i=2}^{\infty} L\left(\partial D_{i}\right)^{2}+2 L\left(\partial D_{1}\right) \sum_{i=2}^{\infty} L\left(\partial D_{i}\right) \\
& \geq 4 \pi\left(\sum_{i=1}^{\infty} x_{i}+2 \sqrt{x_{1}} \sum_{i=2}^{\infty} \sqrt{x_{i}}\right) \\
& \geq 4 \pi\left(A(D)+2 \sqrt{x_{1}}\left(\sum_{i=2}^{\infty} x_{i}\right)^{1 / 2}\right) \\
& =4 \pi\left(A(D)+2 \sqrt{x_{1}\left(A(D)-x_{1}\right)}\right) .
\end{aligned}
$$

Recalling that $\delta A(D) \leq x_{1} \leq(1-\delta) A(D)$, and using the fact that $x(1-x)$ for $x \in[\delta, 1-\delta]$ has as its minimum $\delta(1-\delta)$, we have

$$
L(\partial D)^{2} \geq 4 \pi(1+\sqrt{\delta}) A(D) .
$$


Thus the statement of the proposition holds in this case.

We now consider the case when $x_{1}$ is small, i.e., $x_{1}<\delta A(D)$. Then

$$
\delta A(D)>x_{1} \geq x_{2} \geq x_{3} \geq \cdots
$$

and

$$
\sum_{i \neq \ell} x_{i} \geq(1-\delta) A(D), \quad \text { for all } \ell=1,2, \ldots
$$

Clearly,

$$
\begin{aligned}
L(\partial D)^{2} & =\left(\sum_{i=1}^{\infty} L\left(\partial D_{i}\right)\right)^{2} \\
& =\left(\sum_{i=1}^{\infty} L\left(\partial D_{i}\right)^{2}+\sum_{j=1}^{\infty} L\left(\partial D_{j}\right) \sum_{i \neq j} L\left(\partial D_{i}\right)\right) \\
& \geq 4 \pi\left(A(D)+\sum_{j=1}^{\infty} \sqrt{x_{j}} \sum_{i \neq j} \sqrt{x_{i}}\right) .
\end{aligned}
$$

Setting $\varepsilon_{i}=x_{i} / x_{1} \leq 1$, and employing (2.6), we obtain

$$
\begin{aligned}
\sum_{j=1}^{\infty} \sqrt{x_{j}} \sum_{i \neq j} \sqrt{x_{i}} & =x_{1}\left(\sum_{j=1}^{\infty} \sqrt{\varepsilon_{j}} \sum_{i \neq j} \sqrt{\varepsilon_{i}}\right) \\
& \geq x_{1}\left(\sum_{j=1}^{\infty} \varepsilon_{j} \sum_{i \neq j} \varepsilon_{i}\right) \\
& \geq \frac{(1-\delta) A(D)^{2}}{x_{1}} \\
& \geq \frac{(1-\delta)}{\delta} A(D) .
\end{aligned}
$$

The proposition now follows easily in this second case by combining (2.7) and (2.8).

By taking the contrapositive of Proposition 2.1, we have

Proposition 2.2. Let $D$ be a bounded open set such that, for some $\delta$ $(0<\delta<1 / 4), L(\partial D)$ satisfies

$$
L(\partial D)^{2}<4 \pi(1+\sqrt{\delta}) A(D) .
$$


If $D_{1}$ is a component of $D$ with the largest area, then

$$
A\left(D_{1}\right)>(1-\delta) A(D) .
$$

REMARK 2.1. The exponent $1 / 2$ appearing on $\delta$ in the statement of Proposition 2.1 is sharp. To see this take $D=D_{1} \cup D_{2}$, where $D_{1}$ and $D_{2}$ are two disjoint discs of radius $\sqrt{1-\delta}$ and $\sqrt{\delta}$ respectively. Take $\delta<1 / 4$. Then $A(D)=\pi$, and $A\left(D_{1}\right)=(1-\delta) A(D)$. Clearly, $L(\partial D)^{2}=4 \pi(1+O(\sqrt{\delta})) A(D)$, as $\delta \rightarrow 0$.

For a condenser $\Gamma$ with inner set $\Omega$ and outer set $\mathbb{R}^{2} \backslash \Omega^{\prime}$, if $u$ is the extremal extended to be zero on $\Omega$, we write $F(t)=\{x: u(x)<t\}$ and $A(t)=A(F(t))(0<t \leq 1)$. We will often write $\alpha=\alpha(\Omega)$ for convenience.

Our proof of Theorem 1 will be broken down into two cases. In Case 1 , the asymmetry of $\Omega$ is propagated through a $t$ interval for the sets $F(t)$. Here the proof follows the methods of [6]. It is easy to construct examples of sets $\Omega$ for which $\alpha(F(t))$ is dramatically less than $\alpha(\Omega)$ for $t$ arbitrarily close to zero. Case 2 is designed to cover this possibility.

The plan in Case 2 is as follows. Since $\alpha(F(T))$ is very small for some $T$ close to 0 , we first observe that this implies that most of $F(T)$ is a set, which we later call $F_{1}$, whose boundary is contained between very close concentric circles. This is the essence of (6.18) below. By using the symmetrization of Section 3, we construct a new condenser with comparable asymmetry and decreased $p$-capacity by suitably redistributing the portion of $F_{1}$ on each ray from the center $x_{0}$ of the concentric circles. Using the new configuration, we then obtain a lower bound on the capacities stated in Lemma 4.1.

In what follows, $\kappa$ and $\eta$ will denote small positive constants which do not depend on $\alpha$, and which will be determined later. We assume

$$
0<\kappa<0.0001, \eta \leq 0.01 \text {, and } \kappa<\frac{\eta^{2}}{10} \text {. }
$$

Case 1. For all $t$ such that

$$
1+\eta \leq A(t) \leq 1+2 \eta
$$

we have

$$
L(\partial F(t))^{2} \geq 4 \pi\left(1+\kappa \alpha^{2}\right) A(t) .
$$


Case 2. There exists a value $T$ such that

$$
1+\eta \leq A(T) \leq 1+2 \eta
$$

and

$$
L(\partial F(T))^{2}<4 \pi\left(1+\kappa \alpha^{2}\right) A(T) .
$$

By the result in [13], in Case $1, D u$ can vanish on at most a finite number of levels $u=t$ in the interval specified by (2.10). In Case 2 , by making a slight adjustment, we may choose $T$ such that $D u$ is nonvanishing on the boundary of $F(T)$. Thus we may take $\partial F(T)$ to be analytic in the latter case.

\section{A symmetrization technique.}

We now present a new type of symmetrization which will be useful in relating $p$-capacity to asymmetry. Let $\Omega_{1}$ and $F_{1}$ be two bounded open subsets of $\mathbb{R}^{2}$. We assume that i) $\bar{\Omega}_{1} \subset F_{1}$, ii) the origin 0 lies in $\Omega_{1}$, and iii) $\partial \Omega_{1}$ and $\partial F_{1}$ are the unions of finitely many Lipschitz continuous curves. Let $\rho=\sqrt{A\left(\Omega_{1}\right) / \pi}$ and $R=\sqrt{A\left(F_{1}\right) / \pi}$.

For each $\theta \in(-\pi, \pi]$, let $J(\theta)=\left\{r e^{i \theta}: 0 \leq r\right\}$ be the ray from the origin making an angle $\theta$ with the positive $x$-axis. For a given value of $\theta$, let

$$
J(\theta) \cap \Omega_{1}=\left[r_{0}, r_{1}(\theta)\right) \bigcup_{j \geq 1}\left(r_{2 j}(\theta), r_{2 j+1}(\theta)\right), \quad r_{0}=0,
$$

the intervals being disjoint. We now introduce the parameters necessary to give a redistribution of the area of $\Omega_{1}$ relative to $B(0, \rho)$. Set

$$
\begin{aligned}
s(\theta) & =\sup \left\{r: r e^{i \theta} \in J(\theta) \cap \Omega_{1}\right\}, \\
t(\theta) & =\inf \left\{r: r e^{i \theta} \in J(\theta) \cap \partial F_{1}\right\} \\
& =\sup \left\{r:[0, r) \subset J(\theta) \cap F_{1}\right\}, \\
\hat{s}(\theta) & =\sup \left\{r: r e^{i \theta} \in J(\theta) \cap \Omega_{1}, r<t(\theta)\right\}, \\
\hat{t}(\theta) & =\inf \left\{r: r e^{i \theta} \in J(\theta) \cap \partial F_{1}, r>s(\theta)\right\} \\
& =\sup \left\{r:[s(\theta), r) \subset J(\theta) \cap F_{1}\right\}, \\
N & =\left\{r e^{i \theta} \in \Omega_{1}: s(\theta)>t(\theta), r>\hat{s}(\theta)\right\}, \\
E & =\{\theta: J(\theta) \cap N \neq \varnothing\} .
\end{aligned}
$$


602 T. Bhattacharya and A. Weitsman

Figure 1. $\hat{s}(\theta)=s(\theta)<t(\theta)=\hat{t}(\theta)$.

Figure 2. $\hat{s}(\theta)<t(\theta)<s(\theta)<\hat{t}(\theta) \quad$ (Shaded Region in $N)$.

Note that $\hat{s}(\theta) \leq s(\theta)$ and $\hat{t}(\theta) \geq t(\theta)$ with equality if and only if $s(\theta)<t(\theta)$.

We distinguish two possibilities in our redistribution of $\Omega_{1}$.

Case A. Suppose first that $\hat{s}(\theta) \leq \rho$. Then we define $\xi(\theta)>0$ by

$$
\xi(\theta)^{2}=\sum_{j \in K} r_{2 j+1}^{2}-r_{2 j}^{2}
$$


where $K=\left\{j: r_{2 j+1} \leq \hat{s}(\theta)\right\}$.

Case B. If $\hat{s}(\theta)>\rho$ we distinguish two subcases to define $\xi(\theta)>0$ and $\lambda(\theta)>0$.

i) If $\rho \in J(\theta) \cap \Omega_{1}$, i.e., $r_{2 m}<\rho<r_{2 m+1}$ for some $m$, then

$$
\xi(\theta)^{2}=r_{2 m+1}^{2}+\sum_{j \in L} r_{2 j+1}^{2}-r_{2 j}^{2}
$$

where $L=\left\{j: r_{2 m+1} \leq r_{2 j}<r_{2 j+1} \leq \hat{s}(\theta)\right\}$; also let

$$
\lambda(\theta)^{2}=\rho^{2}-r_{2 m}^{2}+\sum_{j \in M} r_{2 j+1}^{2}-r_{2 j}^{2}
$$

where $M=\left\{j: r_{2 j+1} \leq r_{2 m}\right\}$.

ii) If $\rho \notin J(\theta) \cap \Omega_{1}$, we set

$$
\xi(\theta)^{2}=\rho^{2}+\sum_{j \in L^{\prime}} r_{2 j+1}^{2}-r_{2 j}^{2}
$$

where $L^{\prime}=\left\{j: \rho \leq r_{2 j}<r_{2 j+1} \leq \hat{s}(\theta)\right\}$, and

$$
\lambda(\theta)^{2}=\sum_{j \in M^{\prime}} r_{2 j+1}^{2}-r_{2 j}^{2}
$$

where $M^{\prime}=\left\{j: r_{2 j+1} \leq \rho\right\}$. It is useful to observe that $\xi(\theta)>\rho$ and $\lambda(\theta) \leq \rho$, whenever $\hat{s}(\theta)>\rho$.

For each $\theta \in(-\pi, \pi]$, let $\Omega_{1}^{*}(\theta) \subset J(\theta)$ be defined by

$$
\Omega_{1}^{*}(\theta)= \begin{cases}{[0, \xi(\theta)],} & \text { if } \hat{s}(\theta) \leq \rho, \\ {[0, \lambda(\theta)] \cup(\rho, \xi(\theta)],} & \text { if } \hat{s}(\theta)>\rho \text { and } \lambda(\theta)<\rho, \\ {[0, \xi(\theta)],} & \text { if } \hat{s}(\theta)>\rho \text { and } \lambda(\theta)=\rho .\end{cases}
$$

Define $\Omega_{1}^{*}=\cup_{\theta} \Omega_{1}^{*}(\theta)$; by the definitions in (3.1)-(3.6), it is clear that i) $A\left(\Omega_{1}\right)=A\left(\Omega_{1}^{*}\right)+A(N)$ (see $(3.10)$ ), ii) if $B(0, r) \subset \Omega_{1}$, then $B(0, r) \subset$ $\Omega_{1}^{*}$, and iii) $\Omega_{1}^{*} \cap B(0, \rho)$ is starlike with respect to the origin 0 .

Now suppose that $0<R_{i}^{\prime} \leq \rho$ and $R_{i} \leq R_{o}$ are such that $\bar{B}\left(0, R_{i}^{\prime}\right)$ $\subset \Omega_{1}$, and $\bar{B}\left(0, R_{i}\right) \subset F_{1} \subset \bar{F}_{1} \subset B\left(0, R_{o}\right)$. Then we conclude from 
(3.1)-(3.6) that
i) $R_{i}^{\prime} \leq \xi(\theta) \leq \hat{s}(\theta) \leq s(\theta) \leq \hat{t}(\theta) \leq R_{o}$ ，
ii) $R_{i} \leq t(\theta) \leq \hat{t}(\theta) \leq R_{o}$,
iii) $R_{i} \leq t(\theta)<s(\theta)<\hat{t}(\theta) \leq R_{o}, \theta \in E$,
iv) $R_{i}^{\prime} \leq \xi(\theta)<t(\theta) \leq R_{o}$,
v) $R_{i}^{\prime} \leq \min \left\{\rho, R_{i}\right\} \leq \max \left\{\rho, R_{i}\right\} \leq R \leq R_{o}$ ，
vi) If $\hat{s}(\theta)>\rho$, then $\xi(\theta)>\rho$, and $\lambda(\theta) \leq \rho$.

Based on (3.7) we now make some easy observations. These will be useful in Section 4 and Section 8. Suppose that $\beta=A\left(\Omega_{1} \backslash B(0, \rho)\right) / A\left(\Omega_{1}\right)$ $>0$. By consideration of $\Omega_{1} \backslash B(0, \rho)$ we have

$$
\begin{aligned}
0 & <2 \pi \rho^{2}\left(\beta-\frac{A(N \backslash B(0, \rho))}{\pi \rho^{2}}\right) \\
& =\int_{\{\xi(\theta) \geq \rho\}}\left(\xi(\theta)^{2}-\rho^{2}\right) d \theta \leq 2 \pi \rho^{2} \beta .
\end{aligned}
$$

By consideration of $\Omega_{1} \cap B(0, \rho)$,

$$
\begin{aligned}
0 & <\int_{\{\xi(\theta) \leq \rho\}}\left(\rho^{2}-\xi(\theta)^{2}\right) d \theta+\int_{\{\hat{s}(\theta)>\rho\}}\left(\rho^{2}-\lambda(\theta)^{2}\right) d \theta \\
& =2 \pi \rho^{2}\left(\beta+\frac{A(N \cap B(0, \rho))}{\pi \rho^{2}}\right) .
\end{aligned}
$$

Subtracting (3.8) from (3.9), we then have

$$
0<\int_{-\pi}^{\pi}\left(\rho^{2}-\xi(\theta)^{2}\right) d \theta+\int_{\{\hat{s}(\theta)>\rho\}}\left(\rho^{2}-\lambda(\theta)^{2}\right) d \theta=2 A(N)
$$

and adding we obtain

$$
\begin{aligned}
\int_{-\pi}^{\pi}\left|\rho^{2}-\xi(\theta)^{2}\right| d \theta= & \int_{\{\xi(\theta) \geq \rho\}}\left(\xi(\theta)^{2}-\rho^{2}\right) d \theta \\
& +\int_{\{\xi(\theta) \leq \rho\}}\left(\rho^{2}-\xi(\theta)^{2}\right) d \theta \\
\leq & 4 \pi \rho^{2}\left(\beta+\frac{A(N)}{\pi \rho^{2}}\right)
\end{aligned}
$$


Also, let

$$
\left\{\begin{array}{l}
\mu=\frac{1}{\rho^{2}} \int_{\{\hat{s}(\theta)>\rho\}}\left(\rho^{2}-\lambda(\theta)^{2}\right) d \theta \geq 0, \\
\bar{\mu}=\frac{1}{R^{2}} \int_{-\pi}^{\pi}\left(R^{2}-t(\theta)^{2}\right) d \theta \geq 0 .
\end{array}\right.
$$

In the next section we will use this symmetrization technique to deduce a perturbation result for 2-capacity.

\section{A perturbation lemma for 2-capacity.}

We will now prove a perturbation lemma based on the symmetrization introduced in Section 3. As before, $\Omega_{1}$ and $F_{1}$, subsets of $\mathbb{R}^{2}$, are bounded open sets such that i) $\bar{\Omega}_{1} \subset F_{1}$, ii) the origin 0 lies in $\Omega_{1}$, and iii) $\partial \Omega_{1}$ and $\partial F_{1}$ are the unions of finitely many Lipschitz continuous curves. Set $\rho=\sqrt{A\left(\Omega_{1}\right) / \pi}$ and $R=\sqrt{A\left(F_{1}\right) / \pi}$. Let $0<R_{i}^{\prime} \leq \rho$ and $R_{i} \leq R_{o}$ be such that $\bar{B}\left(0, R_{i}^{\prime}\right) \subset \Omega_{1}, \bar{B}\left(0, R_{i}\right) \subset F_{1} \subset \bar{F}_{1} \subset B\left(0, R_{o}\right)$. Suppose furthermore that

i) For a fixed $\varepsilon, 0<\varepsilon \leq 1 / 2, R_{o}(1-\varepsilon) \leq R_{i} \leq R \leq R_{o}$,

$$
\begin{aligned}
& \text { ii) } 1 / 2 \leq R_{i}^{\prime} / R_{o} \leq R_{i} / R_{o} \leq 1 \text {, } \\
& \text { iii) For } 0<\delta \leq 1 / 2,1 / 4 \leq(\rho / R)^{2} \leq 1 /(1+\delta)<1 \text {. }
\end{aligned}
$$

By the definition in (1.4), if $\Gamma=\Gamma\left(\bar{\Omega}_{1}, \mathbb{R}^{2} \backslash F_{1}\right)$, then

$$
I=\operatorname{Cap}_{2}(\Gamma)=\inf _{w} \int_{F_{1} \backslash \Omega_{1}}|D u|^{2} d x d y
$$

where, $w$ is absolutely continuous and takes the value 1 on $\mathbb{R}^{2} \backslash F_{1}$ and 0 on $\bar{\Omega}_{1}$. Let $v$ denote the minimizer. Then it is harmonic in $F_{1} \backslash \bar{\Omega}_{1}$ and assumes the appropriate boundary values. Set

$$
\beta=\frac{A\left(\Omega_{1} \backslash B(0, \rho)\right)}{A\left(\Omega_{1}\right)}>0
$$

We prove 
Lemma 4.1. Let $\Omega_{1}, F_{1}, \rho, R, R_{i}, R_{i}^{\prime}, R_{o}, \beta, \varepsilon, \delta$, and $v$ be as described above. Assume that (4.1) holds. Then for all sufficiently small $\varepsilon$, we have

$$
I=\int_{F_{1} \backslash \Omega_{1}}|D v|^{2} d x d y \geq \frac{2 \pi}{\log R / \rho}+B_{0} \beta^{2}-B_{1} \varepsilon^{2}-B_{2} \varepsilon \beta,
$$

where $B_{0}, B_{1}$ and $B_{2}$ are positive constants depending only on $\delta$.

Proof. Throughout the proof we shall let $C$, with or without subscripts, denote positive constants depending only on $\delta$, and which need not be the same at each occurrence. We employ the symmetrization introduced in Section 3, and use the same notations as in (3.1)-(3.6). Then from (3.7) and (4.1), we may conclude that
i) $0<\hat{t}(\theta)-s(\theta) \leq \varepsilon R_{o}, \theta \in E$,
ii) $(1 / e)^{2}<(1 / 2)^{2} \leq \min \left\{\xi(\theta)^{2} / R^{2}, \xi(\theta)^{2} / t(\theta)^{2}\right\}$,
iii) $\left|R^{2}-t(\theta)^{2}\right| \leq 2 \varepsilon R_{o}^{2}$.
iv) $1-\varepsilon \leq t(\theta) / R_{o} \leq 1$.

Now

$$
\begin{aligned}
I & =\int_{F_{1} \backslash \Omega_{1}}\left(v_{r}^{2}+\frac{1}{r^{2}} v_{\theta}^{2}\right) r d r d \theta \\
& \geq \int_{F_{1} \backslash \Omega_{1}} v_{r}^{2} r d r d \theta \\
& \geq \int_{-\pi}^{\pi}\left(\inf \int_{J(\theta) \cap\left\{F_{1} \backslash \Omega_{1}\right\}} z_{r}^{2} r d r\right) d \theta,
\end{aligned}
$$

where the infimum is taken over all $z=z(r, \theta)$ such that $z=1$ on $J(\theta) \cap$ $\partial F_{1}$ and $z=0$ on $J(\theta) \cap \partial \Omega_{1}$. The minimizer $\bar{z}$ satisfies the one variable Euler equation $\left(r \bar{z}^{\prime}\right)^{\prime}=0$ in $J(\theta) \cap\left\{F_{1} \backslash \bar{\Omega}_{1}\right\}$. We will now estimate $I$ by employing the symmetrization in Section 3 and obtaining a lower bound for the inner integral on the right side of (4.3). We do this by first solving for $\bar{z}$ from the aforementioned o.d.e over the disjoint intervals $(\hat{s}(\theta), t(\theta))$ and $(s(\theta), \hat{t}(\theta))$, the latter occurring whenever $s(\theta)>t(\theta)$. Note that $\bar{z}$ vanishes on the left end points of these intervals and takes the value 1 on the right end points. Also see (3.7). Thus a lower bound for $I$ is obtained by calculating the inner integral for this function $\bar{z}$ 
over the above mentioned intervals. Recalling the definition of $E$ from (3.1), it follows from (4.3), (3.7.i)), and (3.1) that

$$
\begin{aligned}
I & \geq \int_{-\pi}^{\pi} \frac{1}{\log (t(\theta) / \hat{s}(\theta))} d \theta+\int_{E} \frac{1}{\log (\hat{t}(\theta) / s(\theta))} d \theta \\
& \geq \int_{-\pi}^{\pi} \frac{1}{\log (t(\theta) / \xi(\theta))} d \theta+\int_{E} \frac{1}{\log (\hat{t}(\theta) / s(\theta))} d \theta .
\end{aligned}
$$

If the second integral, on the right hand side of (4.4), is larger than $4 \pi / \log (R / \rho)$ then Lemma 4.1 follows trivially from (4.1.iii)). Otherwise,

$$
\int_{E} \frac{1}{\log (\hat{t}(\theta) / s(\theta))} d \theta \leq \frac{4 \pi}{\log (R / \rho)}
$$

But, $\log (\hat{t}(\theta) / s(\theta)) \leq(\hat{t}(\theta) / s(\theta)-1)$, so it then follows from (4.2.i)), (4.1.ii)-iii)) and (3.7.iii)) that

$$
\operatorname{meas}_{\theta} E \leq C_{1} \varepsilon \text {. }
$$

Note that $C_{1}$ depends only on $\delta$. Since

$$
N=\left\{r e^{i \theta} \in \Omega_{1}: s(\theta)>t(\theta), r>\hat{s}(\theta)\right\},
$$

(4.1.i)) then yields

$$
A(N) \leq C_{2} \varepsilon^{2} R_{o}^{2}
$$

Now, from (4.4),

$$
\begin{aligned}
I & \geq \int_{-\pi}^{\pi} \frac{1}{\log (t(\theta) / \xi(\theta))} d \theta \\
& =2 \int_{-\pi}^{\pi} \frac{-1}{\log \left(\xi(\theta)^{2} / t(\theta)^{2}\right)} d \theta .
\end{aligned}
$$

To estimate (4.6) we observe that the function $f(x)=-1 / \log x$ satisfies

$$
\begin{cases}\text { i) } & f(x)>0 \quad(0<x<1), \\ \text { ii) } & f^{\prime}(x)>0 \quad(0<x<1), \\ \text { iii) } & f^{\prime \prime}(x)>0 \quad\left(1 / e^{2}<x<1\right) .\end{cases}
$$


We shall use (4.7) in the form

$$
f(x)-f(\bar{x})=f(\bar{x})(x-\bar{x})+\frac{f^{\prime \prime}(\zeta)}{2}(x-\bar{x})^{2}
$$

for some $\zeta \in(x, \bar{x})$ or $(\bar{x}, x)$. Then with $\bar{x}=\rho^{2} / R^{2}$, it follows from (4.1), (4.2), (4.6), (4.7) and (4.8) that

$$
\begin{aligned}
I-\frac{2 \pi}{\log (R / \rho)} \geq & 2 \int_{-\pi}^{\pi}\left(\frac{-1}{\log \left(\xi(\theta)^{2} / t(\theta)^{2}\right)}+\frac{1}{\log \left(\rho^{2} / R^{2}\right)}\right) d \theta \\
\geq & 2 f^{\prime}\left(\rho^{2} / R^{2}\right) \int_{-\pi}^{\pi}\left(\frac{\xi(\theta)^{2}}{t(\theta)^{2}}-\frac{\rho^{2}}{R^{2}}\right) d \theta \\
& +C_{3} \int_{-\pi}^{\pi}\left(\frac{\xi(\theta)^{2}}{t(\theta)^{2}}-\frac{\rho^{2}}{R^{2}}\right)^{2} d \theta .
\end{aligned}
$$

The positive absolute constant $C_{3}$ in (4.9) results from the fact that (4.2.ii)) implies that $\xi(\theta)^{2} / t(\theta)^{2}>1 / e^{2}$.

Next we estimate the quantities

$$
S=\int_{-\pi}^{\pi}\left(\frac{\xi(\theta)^{2}}{t(\theta)^{2}}-\frac{\rho^{2}}{R^{2}}\right) d \theta, \quad \bar{S}=\int_{-\pi}^{\pi}\left(\frac{\xi(\theta)^{2}}{t(\theta)^{2}}-\frac{\rho^{2}}{R^{2}}\right)^{2} d \theta .
$$

We may rewrite $S$ as

$S=\int_{-\pi}^{\pi}\left(\left(\xi(\theta)^{2}-\rho^{2}\right)\left(\frac{1}{t(\theta)^{2}}-\frac{1}{R^{2}}\right)+\rho^{2}\left(\frac{1}{t(\theta)^{2}}-\frac{1}{R^{2}}\right)+\frac{\xi(\theta)^{2}-\rho^{2}}{R^{2}}\right) d \theta$.

By (3.10) and (3.12)

$$
\int_{-\pi}^{\pi} \frac{\xi(\theta)^{2}-\rho^{2}}{R^{2}} d \theta=\frac{\mu \rho^{2}}{R^{2}}-\frac{2 A(N)}{R^{2}} \geq-\frac{2 A(N)}{R^{2}} .
$$

Also, by (3.11), (4.1.ii)-iii)), (4.2.iii-iv)),

$$
\begin{aligned}
\mid \int_{-\pi}^{\pi}\left(\xi(\theta)^{2}-\rho^{2}\right) & \left(\frac{1}{t(\theta)^{2}}-\frac{1}{R^{2}}\right) d \theta \mid \\
& \leq \int_{-\pi}^{\pi}\left|\xi(\theta)^{2}-\rho^{2}\right|\left|\frac{1}{t(\theta)^{2}}-\frac{1}{R^{2}}\right| d \theta \\
& \leq \frac{C_{4} \varepsilon}{R^{2}} \int_{-\pi}^{\pi}\left|\xi(\theta)^{2}-\rho^{2}\right| d \theta \\
& \leq C_{5} \varepsilon\left(\beta+\frac{A(N)}{\pi \rho^{2}}\right) .
\end{aligned}
$$


By (3.12)

$$
\begin{aligned}
\int_{-\pi}^{\pi}( & \left.\frac{1}{t(\theta)^{2}}-\frac{1}{R^{2}}\right) d \theta \\
\quad & \int_{-\pi}^{\pi}\left(\frac{R^{2}-t(\theta)^{2}}{R^{2} t(\theta)^{2}}-\frac{R^{2}-t(\theta)^{2}}{R^{4}}+\frac{\bar{\mu}}{2 \pi R^{2}}\right) d \theta \\
& =\int_{-\pi}^{\pi}\left(\frac{\left(R^{2}-t(\theta)^{2}\right)^{2}}{R^{4} t(\theta)^{2}}+\frac{\bar{\mu}}{2 \pi R^{2}}\right) d \theta \\
& \geq 0
\end{aligned}
$$

Putting together (4.10), (4.11), and (4.12) we have

$$
S=\int_{-\pi}^{\pi}\left(\frac{\xi(\theta)^{2}}{t(\theta)^{2}}-\frac{\rho^{2}}{R^{2}}\right) d \theta \geq-\frac{2 A(N)}{R^{2}}-C_{5} \varepsilon\left(\beta+\frac{A(N)}{\pi \rho^{2}}\right)
$$

We now estimate $\bar{S}$. Observe that

$$
\frac{1}{2}\left(\frac{\xi(\theta)^{2}}{R^{2}}-\frac{\rho^{2}}{R^{2}}\right)^{2} \leq\left(\frac{\xi(\theta)^{2}}{R^{2}}-\frac{\xi(\theta)^{2}}{t(\theta)^{2}}\right)^{2}+\left(\frac{\xi(\theta)^{2}}{t(\theta)^{2}}-\frac{\rho^{2}}{R^{2}}\right)^{2}
$$

Integrating with respect to $\theta$ and recalling (3.7.i)), (4.1.i)-ii)) and (4.2.ii)), we have

$$
\int_{-\pi}^{\pi}\left(\frac{\xi(\theta)^{2}}{R^{2}}-\frac{\xi(\theta)^{2}}{t(\theta)^{2}}\right)^{2} d \theta \leq C_{6} \varepsilon^{2}
$$

Using Hölder's inequality,

$$
\begin{aligned}
\left(\int_{\xi(\theta) \geq \rho}\left(\xi(\theta)^{2}-\rho^{2}\right) d \theta\right)^{2} & \leq\left(\int_{-\pi}^{\pi}\left|\xi(\theta)^{2}-\rho^{2}\right| d \theta\right)^{2} \\
& \leq 2 \pi \int_{-\pi}^{\pi}\left(\xi(\theta)^{2}-\rho^{2}\right)^{2} d \theta
\end{aligned}
$$

so by (3.8) and (4.1.iii)),

$$
\frac{1}{2} \int_{-\pi}^{\pi}\left(\frac{\xi(\theta)^{2}}{R^{2}}-\frac{\rho^{2}}{R^{2}}\right)^{2} d \theta \geq C_{7}\left(\beta-\frac{A(N)}{\pi \rho^{2}}\right)^{2} .
$$

Putting together (4.14) and (4.15) we obtain

$$
\bar{S}=\int_{-\pi}^{\pi}\left(\frac{\xi(\theta)^{2}}{t(\theta)^{2}}-\frac{\rho^{2}}{R^{2}}\right)^{2} d \theta \geq C_{8} \beta^{2}-C_{9} \varepsilon^{2}-C_{10} \frac{A(N)}{\pi \rho^{2}}
$$


By virtue of (4.1) and (4.2), the positive constants $C_{1}-C_{10}$ depend only on $\delta$. The estimates in (4.13), (4.16) and (4.5) in (4.9) then give

$$
I \geq \frac{2 \pi}{\log (R / \rho)}+B_{0} \beta^{2}-B_{1} \varepsilon^{2}-B_{2} \varepsilon \beta .
$$

where $B_{0}, B_{1}$, and $B_{2}$ are positive constants depending only on $\delta$. This concludes the proof of Lemma 4.1.

A $p$-analogue of Lemma 4.1 appears in Section 8 .

REMARK 4.1. The constants $B_{0}, B_{1}$ and $B_{2}$ appearing in the statement of the Lemma 4.1, become absolute once a numerical value for $\delta$ is chosen. In our application of Lemma 4.1, a positive value for $\delta$ will be fixed once a positive value for $\eta$, appearing in (2.9)-(2.13), is chosen. In particular, we may take $\delta=0.9 \eta$. See $(6.29 . \mathrm{x}))$.

In the next four sections, we will present the proof of Theorem 1 , based on the strategy outlined in Section 2. The proof in Case 1 appears in Section 5, while the proof in Case 2 will be presented in sections 6,7 and 8 .

\section{Proof of (1.5) in Case 1.}

We will first prove Theorem 1 in the situation when asymmetry propagates, that is, when (2.10) implies (2.11). It is easy to see that $A(t)$ is continuous and increasing. If we set

$$
s_{0}=\inf \{t \in[0,1]: A(t) \geq 1+\eta\}
$$

and

$$
T_{0}=\sup \{t \in[0,1]: A(t) \leq 1+2 \eta\},
$$

then

$$
A\left(s_{0}\right) \leq A(t) \leq A\left(T_{0}\right), \quad t \in\left[s_{0}, T_{0}\right] .
$$

Recall from Section 1 that $u$ is locally $C^{1, \gamma}$. Hence an application of the coarea formula [2, p. 248] yields, for almost everywhere $t$,

$$
A^{\prime}(t)=\int_{\partial F(t)} \frac{1}{|D u|} d \sigma .
$$


The formula in (5.4) holds except for possibly a discrete set of $t$ 's since the set of critical points of $u$ is discrete. We now prove

Lemma 5.1. Let $1<p<\infty$. If $u$ is the extremal for the condenser with inner set $\Omega$ and outer set $\mathbb{R}^{2} \backslash \Omega^{\prime}$ and $T_{0}$ is as in (5.2), then

$$
\begin{aligned}
T_{0} \leq & \left(\int_{F\left(T_{0}\right)}|D u|^{p} d x d y\right)^{1 / p} \\
& \cdot\left(\frac{1}{1+C \alpha^{2}} \int_{1}^{1+2 \eta} \phi(t) d t\right)^{(p-1) / p},
\end{aligned}
$$

where $\phi(t)=\phi_{p}(t)=(4 \pi t)^{p / 2(1-p)}, \alpha=\alpha(\Omega)$, and $C$ is a constant which depends only on $\kappa, \eta$ and $p$.

Proof. By the coarea formula and (5.4) we have outside a discrete set of $t$ 's,

$$
\begin{aligned}
\int_{\partial F(t)} 1 d \sigma & \leq\left(\int_{\partial F(t)}|D u|^{p-1} d \sigma\right)^{1 / p}\left(\int_{\partial F(t)} \frac{1}{|D u|} d \sigma\right)^{(p-1) / p} \\
& =\left(\int_{\partial F(t)}|D u|^{p-1} d \sigma\right)^{1 / p}\left(A^{\prime}(t)\right)^{(p-1) / p}
\end{aligned}
$$

Using (2.10) and (2.11) it follows, for almost everywhere $t$ with $s_{0}<$ $t \leq T_{0}$ (see (5.1)-(5.3)),

$$
1 \leq\left(\int_{\partial F(t)}|D u|^{p-1} d \sigma\right)^{1 / p}\left(\frac{\left(A^{\prime}(t)\right)^{(p-1) / p}}{\sqrt{4 \pi\left(1+\kappa \alpha^{2}\right) A(t)}}\right) .
$$

We now integrate (5.6) from $s_{0}$ to $T_{0}$. An application of Hölder's inequality then yields

$$
\begin{aligned}
T_{0}-s_{0} \leq & \int_{s_{0}}^{T_{0}}\left(\int_{\partial F(t)}|D u|^{p-1} d \sigma\right)^{1 / p}\left(\frac{\left(A^{\prime}(t)\right)^{(p-1) / p}}{\sqrt{4 \pi\left(1+\kappa \alpha^{2}\right) A(t)}}\right) d t \\
\leq & \left(\int_{s_{0}}^{T_{0}}\left(\int_{\partial F(t)}|D u|^{p-1} d \sigma\right) d t\right)^{1 / p} \\
& \cdot\left(\int_{s_{0}}^{T_{0}} \frac{A^{\prime}(t)}{\left(4 \pi\left(1+\kappa \alpha^{2}\right) A(t)\right)^{p / 2(p-1)}} d t\right)^{(p-1) / p} .
\end{aligned}
$$


Thus, by the coarea formula and the formula for $\phi$ as described in (1.6), we have

$$
\begin{aligned}
T_{0}-s_{0} \leq & \left(\int_{F\left(T_{0}\right) \backslash F\left(s_{0}\right)}|D u|^{p} d x d y\right)^{1 / p} \\
& \cdot\left(\frac{1}{\sqrt{1+\kappa \alpha^{2}}}\left(\int_{1+\eta}^{1+2 \eta} \phi(t) d t\right)^{(p-1) / p}\right) .
\end{aligned}
$$

Using the same procedure on $\left(0, s_{0}\right)$ and the usual isoperimetric inequality in place of (2.11), we see that

$$
s_{0} \leq\left(\int_{F\left(s_{0}\right)}|D u|^{p} d x d y\right)^{1 / p}\left(\int_{1}^{1+\eta} \phi(t) d t\right)^{(p-1) / p}
$$

Adding (5.8) and (5.9) and applying the Hölder inequality, we may show that

$$
\begin{aligned}
T_{0} \leq & \left(\int_{F\left(T_{0}\right)}|D u|^{p} d x d y\right)^{1 / p} \\
& \cdot\left(\int_{1}^{1+\eta} \phi(t) d t+\left(\frac{1}{1+\kappa \alpha^{2}}\right)^{p / 2(p-1)} \int_{1+\eta}^{1+2 \eta} \phi(t) d t\right)^{(p-1) / p} \\
= & \left(\int_{F\left(T_{0}\right)}|D u|^{p} d x d y\right)^{1 / p} \\
& \cdot\left(1-\left(1-\left(\frac{1}{1+\kappa \alpha^{2}}\right)^{p / 2(p-1)}\right) \frac{\int_{1+\eta}^{1+2 \eta} \phi(t) d t}{\int_{1}^{1+2 \eta} \phi(t) d t}\right)^{(p-1) / p} \\
& \cdot\left(\int_{1}^{1+2 \eta} \phi(t) d t\right)^{(p-1) / p} \cdot
\end{aligned}
$$

The inequality in the lemma now follows with an appropriate constant $C=C(\kappa, \eta, p)$. 
ProOf of (1.5) In CASE 1. Using the usual isoperimetric inequality and the above procedure, we may show that

$$
\begin{aligned}
1-T_{0} \leq & \left(\int_{F(1) \backslash F\left(T_{0}\right)}|D u|^{p} d x d y\right)^{1 / p} \\
& \cdot\left(\int_{1+2 \eta}^{4} \phi(t) d t\right)^{(p-1) / p} .
\end{aligned}
$$

We now add (5.5) and (5.10), and then use the Hölder inequality to deduce that

$$
\begin{aligned}
1 \leq & \left(\int_{F(1)}|D u|^{p} d x d y\right)^{1 / p} \\
& \cdot\left(\frac{1}{1+C \alpha^{2}} \int_{1}^{1+2 \eta} \phi(t) d t+\int_{1+2 \eta}^{4} \phi(t) d t\right)^{(p-1) / p} \\
= & \left(\int_{F(1)}|D u|^{p} d x d y\right)^{1 / p}\left(1-\frac{C \alpha^{2}}{1+C \alpha^{2}} \frac{\int_{1}^{1+2 \eta} \phi(t) d t}{\int_{1}^{4} \phi(t) d t}\right)^{(p-1) / p} \\
& \cdot\left(\int_{1}^{4} \phi(t) d t\right)^{(p-1) / p} .
\end{aligned}
$$

Noting (1.6) we easily obtain the statement of Theorem 1.

\section{Geometry of the Sets in Case 2 .}

Assume Case 2 holds. In this section we shall use (2.12) and (2.13) to construct a subcondenser whose inner set is close to a disc. Lemma 4.1 will then provide the necessary estimates for obtaining the 2-capacity of the original condenser.

We may assume, as in $[8$, p. 5], that the components of $\Omega$ are simply connected, so that by the maximum principle, the components of the set $F(t)$ for each $t$ in $(0,1]$, are simply connected. Let $F_{1}(t)$ be one having largest area, and $F_{2}(t)=F(t) \backslash F_{1}(t)$. We first show that it suffices to assume that for some $t$ such that

$$
A(t)<1+\kappa \alpha^{2},
$$


we have

$$
A\left(F_{1}(t)\right)>(1-\eta / 10) A(t)
$$

and

$$
L\left(\partial F_{1}(t)\right)^{2}<4 \pi(1+\eta) A\left(F_{1}(t)\right) .
$$

Let $\tau=\sup \left\{t: A(t)<1+k \alpha^{2}\right\}$. Suppose that (6.2) were false for all $t$ such that $0<t \leq \tau$. It follows from Proposition 2.1 and (2.9) that

$$
L(\partial F(t))^{2} \geq 4 \pi(1+\sqrt{\eta / 10}) A(t), \quad 0<t \leq \tau .
$$

If, on the other hand, (6.2) holds but (6.3) does not, then instead of (6.4) we get

$$
\begin{aligned}
L(\partial F(t))^{2} & \geq L\left(\partial F_{1}(t)\right)^{2} \\
& \geq 4 \pi(1+\eta) A\left(F_{1}(t)\right) \\
& \geq 4 \pi(1+\eta)(1-\eta / 10) A(t) \\
& \geq 4 \pi(1+4 \eta / 5) A(t) .
\end{aligned}
$$

Since the right hand side of (6.4) is greater than that of (6.5) for $\eta<$ 0.01 , we find that if $(6.2)$ or $(6.3)$ were to fail, then at least (6.5) would hold.

If we were to repeat the steps in Lemma 5.1 leading to (5.8) we would get

$$
\begin{aligned}
\tau \leq & \left(\int_{F(\tau)}|D u|^{p} d x d y\right)^{1 / p} \\
& \cdot\left(\frac{1}{\sqrt{1+4 \eta / 5}}\left(\int_{1}^{1+\kappa \alpha^{2}} \phi(t) d t\right)^{(p-1) / p}\right) .
\end{aligned}
$$

Also, corresponding to (5.10) we would have

(6.7) $1-\tau \leq\left(\int_{F(1) \backslash F(\tau)}|D u|^{p} d x d y\right)^{1 / p}\left(\int_{1+\kappa \alpha^{2}}^{4} \phi(t) d t\right)^{(p-1) / p}$ 
Adding (6.6) and (6.7) we would obtain

$$
\begin{aligned}
1 \leq & \left(\int_{F(1)}|D u|^{p} d x d y\right)^{1 / p} \\
& \cdot\left(1-\left(1-\left(\frac{1}{1+4 \eta / 5}\right)^{p / 2(p-1)}\right) \frac{\int_{1}^{1+\kappa \alpha^{2}} \phi(t) d t}{\int_{1}^{4} \phi(t) d t}\right)^{(p-1) / p} \\
& \cdot\left(\int_{1}^{4} \phi(t) d t\right)^{(p-1) / p} .
\end{aligned}
$$

It is easy to see that (1.5) follows for an appropriate constant $K=$ $K(\kappa, \eta, p)$.

Thus we may assume the existence of $t=t_{0}$ such that (6.1)-(6.3) hold. Then $F\left(t_{0}\right)$ has a simply connected component $F_{1}\left(t_{0}\right)$ such that (6.1)-(6.3) become

$$
\begin{gathered}
1<A\left(t_{0}\right)<1+\kappa \alpha^{2}, \\
A\left(F_{1}\left(t_{0}\right)\right)>(1-\eta / 10) A\left(t_{0}\right),
\end{gathered}
$$

and

$$
L\left(\partial F_{1}\left(t_{0}\right)\right)^{2}<4 \pi(1+\eta) A\left(F_{1}\left(t_{0}\right)\right) .
$$

Now, with $T$ as in (2.12) and (2.13), $F_{1}(T)$ is a component of $F(T)$ having largest area and $F_{2}(T)=F(T) \backslash F_{1}(T)$. From (2.9) and (6.8), $T>t_{0}$ and $F(T)$ contains $F\left(t_{0}\right)$. From (2.13) and Proposition 2.2, it follows easily that

$$
A\left(F_{1}(T)\right) \geq\left(1-\kappa^{2} \alpha^{4}\right) A(T) .
$$

It is clear from (6.11) that $A\left(F_{2}(T)\right) \leq \kappa^{2} \alpha^{4} A(T)$. From (6.8), (6.9), (2.9) and (2.12) it follows that $F_{1}\left(t_{0}\right)$ cannot be completely contained in $F_{2}(T)$. Now, since $F_{1}\left(t_{0}\right)$ and $F_{1}(T)$ are both connected and $F_{1}\left(t_{0}\right) \subseteq$ $F(T)$, it follows that

$$
F_{1}\left(t_{0}\right) \subseteq F_{1}(T) \text { and } A\left(F_{2}(T)\right)<\kappa^{2} \alpha^{4} A(T) .
$$


Let $\Omega_{1}=F_{1}(T) \cap F\left(t_{0}\right)$. Then the set $F\left(t_{0}\right) \backslash \Omega_{1}$ is contained in $F_{2}(T)$. From (2.12) and (6.12) we have

$$
A\left(F\left(t_{0}\right) \backslash \Omega_{1}\right) \leq A\left(F_{2}(T)\right) \leq \kappa^{2} \alpha^{4} A(T) \leq 4 \kappa^{2} \alpha^{4} \leq \kappa \alpha^{2} .
$$

Hence,

$$
A\left(\Omega_{1}\right) \geq A\left(t_{0}\right)-\kappa \alpha^{2} \geq 1-\kappa \alpha^{2} .
$$

Based on (6.8)-(6.11) we now form an auxiliary condenser with some observations on the geometry of the sets.

Now, by (2.2), $\partial F_{1}(T)$ lies between two circles $C_{o}=\left\{x:\left|x-x_{o}\right|=\right.$ $\left.R_{o}\right\}$ and $C_{i}=\left\{x:\left|x-x_{i}\right|=R_{i}\right\}, R_{o}>R_{i}$, where by (2.12), (2.13) and (6.11),

$$
\begin{aligned}
R_{o}-R_{i} & \leq \frac{1}{\pi} \sqrt{L\left(\partial F_{1}(T)\right)^{2}-4 \pi A\left(F_{1}(T)\right)} \\
& \leq \frac{1}{\pi} \sqrt{L(\partial F(T))^{2}-4 \pi\left(1-\kappa^{2} \alpha^{4}\right) A(T)} \\
& \leq \frac{1}{\pi} \sqrt{4 \pi\left[\left(1+\kappa \alpha^{2}\right)-\left(1-\kappa^{2} \alpha^{4}\right)\right] A(T)} \\
& \leq 2 \sqrt{\kappa} \alpha .
\end{aligned}
$$

In particular, the centers of $C_{o}$ and $C_{i}$ satisfy

$$
\left|x_{o}-x_{i}\right| \leq 2 \sqrt{\kappa} \alpha \text {. }
$$

Also, by (2.3), (2.9), (2.12), (2.13) and (6.11),

$$
\begin{aligned}
R_{o} & \leq \frac{1}{2 \pi}\left(L\left(\partial F_{1}(T)\right)+\sqrt{L\left(\partial F_{1}(T)\right)^{2}-4 \pi A\left(F_{1}(T)\right)}\right) \\
& \leq \frac{1}{2 \pi}\left(L(\partial F(T))+\sqrt{L(\partial F(T))^{2}-4 \pi\left(1-\kappa^{2} \alpha^{4}\right) A(T)}\right) \\
& \leq \sqrt{\frac{A(T)}{\pi}}\left(\sqrt{1+\kappa \alpha^{2}}+\sqrt{\kappa \alpha^{2}+\kappa^{2} \alpha^{4}}\right) \\
& \leq \sqrt{\frac{1+2 \eta}{\pi}}(1+3 \sqrt{\kappa} \alpha) .
\end{aligned}
$$

Regarding the position of $F_{1}\left(t_{0}\right)$ in $F_{1}(T)$, we note that $(6.8),(6.9)$, (6.10) and (2.4) imply that $F_{1}\left(t_{0}\right)$ contains a disc $B\left(\bar{x}, \bar{R}_{i}\right)$ where

$$
\begin{aligned}
\bar{R}_{i} & \geq(1-\sqrt{\eta}) \sqrt{1-\eta / 10} \sqrt{\frac{A\left(t_{0}\right)}{\pi}} \\
& \geq(1-1.1 \sqrt{\eta}) \sqrt{\frac{A\left(t_{0}\right)}{\pi}} \\
& \geq \frac{1-1.1 \sqrt{\eta}}{\sqrt{\pi}} .
\end{aligned}
$$


Recalling that $\Omega_{1}=F_{1}(T) \cap F\left(t_{0}\right)$ and comparing (6.12)-(6.17) we conclude that

$$
\begin{cases}\text { i) } & B\left(x_{o}, R_{o}\right) \supseteq F_{1}(T) \\ \text { ii) } & B\left(x_{o}, R_{o}(1-\varepsilon)\right) \subseteq F_{1}(T), \quad \varepsilon=7.5 \sqrt{\kappa} \alpha \\ \text { iii) } & B\left(x_{o}, R_{i}^{\prime}\right) \subseteq \Omega_{1}\end{cases}
$$

where

$$
\sqrt{\frac{A\left(F_{1}(T)\right)}{\pi}}-2 \sqrt{\kappa} \alpha \leq R_{i} \leq R_{o} \leq \sqrt{1+2 \eta} \frac{1+3 \sqrt{\kappa} \alpha}{\sqrt{\pi}}
$$

and

$$
R_{i}^{\prime}=2 \bar{R}_{i}-R_{o} \geq \frac{1-0.2 \eta-3(1+2 \eta) \sqrt{\kappa} \alpha}{\sqrt{\pi}}
$$

By (6.8), (6.11), (6.13), and (2.12)

$$
\begin{cases}\text { i) } \quad & 1-\kappa \alpha^{2} \leq A\left(\Omega_{1}\right) \leq 1+\kappa \alpha^{2} \\ \text { ii) } \quad\left(1-\kappa^{2} \alpha^{4}\right) A(T) \leq A\left(F_{1}(T)\right) \leq A(T) \\ \text { iii) } \quad 1+\eta \leq A(T) \leq 1+2 \eta\end{cases}
$$

It follows from (2.9) and (6.21) that

$$
1+0.9 \eta \leq \frac{A\left(F_{1}(T)\right)}{A\left(\Omega_{1}\right)} \leq 1+2.1 \eta .
$$

If $B\left(x_{o}, \rho\right)$ has the same area as $\Omega_{1}$ and $B(\tilde{x}, \sqrt{1 / \pi})$ is such that $\alpha=$ $A(\Omega \backslash B(\tilde{x}, \sqrt{1 / \pi}))$, then by $(1.1),(6.18)$ and $(6.21)$

$$
\begin{aligned}
A\left(\Omega_{1} \backslash B\left(x_{o}, \rho\right)\right) \geq & A\left(\Omega \backslash B\left(x_{o}, \rho\right)\right)-A\left(\Omega \backslash \Omega_{1}\right) \\
\geq & A\left(\Omega \backslash B\left(x_{o}, r\right)\right) \\
& -A\left(B\left(x_{o}, \rho\right) \backslash B\left(x_{o}, r\right)\right)-A\left(\Omega \backslash \Omega_{1}\right) \\
\geq & A(\Omega \backslash B(\tilde{x}, r)) \\
& -A\left(B\left(x_{o}, \rho\right) \backslash B\left(x_{o}, r\right)\right)-A\left(\Omega \backslash \Omega_{1}\right) \\
\geq & \alpha-\kappa \alpha^{2}-\kappa \alpha^{2} \\
> & \frac{\alpha}{2}
\end{aligned}
$$


where $r=\sqrt{1 / \pi}$. The third inequality follows from the definition of $\alpha(\Omega)$. Thus, if

$$
\beta=\frac{A\left(\Omega_{1} \backslash B\left(x_{o}, \rho\right)\right)}{A\left(\Omega_{1}\right)}, \quad \rho=\sqrt{\frac{A\left(\Omega_{1}\right)}{\pi}},
$$

we have, from (2.9), (6.23) and (6.21) i) that

$$
\beta>\frac{\alpha}{2\left(1+\kappa \alpha^{2}\right)}>\frac{\alpha}{3} .
$$

We set $F_{1}=F_{1}(T)$ for convenience, and let $u=u_{p}$ be the minimizer for (1.4). Clearly,

$$
\int_{F(T)}|D u|^{p} d x d y \geq \int_{F_{1} \backslash \Omega_{1}}|D u|^{p} d x d y .
$$

Also, since $\partial F_{1}$ and $\partial \Omega_{1}$ are level sets for $u$, we may use $u$, renormalized, as the extremal for the condenser having inner set $\overline{\Omega_{1}}$ (closure of $\Omega$ ) and outer set $\mathbb{R}^{2} \backslash F_{1}$, and in this way estimate the right hand side of (6.26). For $p=2$, this will be done by using Lemma 4.1, while for $p \neq 2$, the $p$-analogue (see Section 8) will be used.

In fact, with $u=t_{0}$ on $\partial \Omega_{1}$ and $u=T$ on $\partial F_{1}$, then

$$
v=\frac{u-t_{0}}{T-t_{0}}
$$

is the minimizer for

$$
\int_{F_{1} \backslash \Omega_{1}}|D w|^{p} d x d y, \quad w= \begin{cases}1, & \text { on } \partial F_{1} \\ 0, & \text { on } \partial \Omega_{1}\end{cases}
$$

Thus,

$$
\begin{aligned}
\inf _{w} \int_{F_{1} \backslash \Omega_{1}}|D w|^{p} d x d y & =\int_{F_{1} \backslash \Omega_{1}}|D v|^{p} d x d y \\
& =\frac{1}{\left(T-t_{0}\right)^{p}} \int_{F_{1} \backslash \Omega_{1}}|D u|^{p} d x d y .
\end{aligned}
$$

Thus, with $\Gamma=\Gamma\left(\bar{\Omega}_{1}, \mathbb{R}^{2} \backslash F_{1}\right)$ as the subcondenser, the next step in the proof of Theorem 1 is to obtain estimates for $\operatorname{Cap}_{p}(\Gamma)$. To this end, we first employ the symmetrization introduced in Section 3. Setting 
$\rho=\sqrt{A\left(\Omega_{1}\right) / \pi}$ and $R=\sqrt{A\left(F_{1}\right) / \pi}$, and using the notations (3.1)(3.6), we conclude from (3.7), (6.14), (6.16), (6.18)-(6.22) that

$$
\begin{cases}\text { i) } \quad & \text { if } \hat{s}(\theta)>\rho, \text { then } \xi(\theta)>\rho \text { and } \lambda(\theta) \leq \rho \\ \text { ii) } \quad & R_{i}^{\prime} \leq \xi(\theta) \leq R_{o} \\ \text { iii }) & R_{o}(1-\varepsilon) \leq R_{i} \leq R \leq R_{o} \\ \text { iv }) \quad & R_{o}(1-\varepsilon) \leq t(\theta) \leq R_{o} \\ \text { v) } \quad R_{i}^{\prime} \leq \rho<R \leq R_{o} \\ \text { vi) } \quad\left|R^{2}-t(\theta)^{2}\right| \leq \varepsilon R_{o}^{2} \\ \text { vii } \quad 0<\hat{t}(\theta)-s(\theta) \leq \varepsilon R_{o} \\ \text { viii) } \quad \xi(\theta) \leq s(\theta) \leq \hat{t}(\theta) \leq R_{o}, \\ \text { ix } \quad R_{i}^{\prime} \leq \xi(\theta)<t(\theta) \leq R_{o} \\ \text { x) } \quad \sqrt{1+0.9 \eta} \leq R / \rho \leq \sqrt{1+2.1 \eta}, \\ \text { xi) } \quad R_{o}(1-\varepsilon) \leq t(\theta)<s(\theta)<\hat{t}(\theta) \leq R_{o}, \quad \theta \in E .\end{cases}
$$

In Section 7 , we will prove Theorem 1 when $p=2$. The details of the proof, when $p \neq 2$, together with the $p$-analogue of Lemma 4.1 will be presented in Section 8 .

\section{Proof of (1.5) for $p=2$ in Case 2.}

We now prove Theorem 1 , in Case 2 , when $p=2$. We specify $\eta=.01$ when $p=2$.

We now take a) $\Omega_{1}=\Omega_{1}\left(t_{0}\right), F_{1}=F_{1}(T), \rho=\sqrt{A\left(\Omega_{1}\right) / \pi}$, and $R=\sqrt{A\left(F_{1}\right) / \pi}$, and b) $R_{i}^{\prime}, R_{i}, R_{o}, \varepsilon$ and $v$ as in (6.20), (6.19), (6.16), (6.18) and (6.27), and c) $x_{o}=0$ in (6.18). As in Remark 4.1, we take $\delta=0.9, \eta=0.009$ (see $(6.29 . \mathrm{x})))$. These observations together with (6.29) imply that the hypotheses of Lemma 4.1 are satisfied. It is easily seen from (6.18) and (6.21) that

$$
\frac{1}{2} \log \frac{A(T)}{1-\kappa \alpha^{2}} \geq \log \frac{R}{\rho} .
$$

We apply the conclusion of Lemma 4.1, together with (6.25)-(6.28), (7.1) and the definition of $\varepsilon$ in (6.18), to conclude that there are absolute constants $C$ and $\kappa_{1}$ such that $\kappa \leq \kappa_{1}$,

$$
\int_{F(T)}|D u|^{2} d x d y \geq \int_{F_{1} \backslash \Omega_{1}}|D u|^{2} d x d y
$$




$$
\begin{gathered}
\geq\left(T-t_{0}\right)^{2}\left(\frac{4 \pi}{\log \left(A(T) /\left(1-\kappa \alpha^{2}\right)\right)}\right. \\
\left.+B_{0} \beta^{2}-B_{1} \varepsilon^{2}-B_{2} \varepsilon \beta\right) \\
\geq\left(T-t_{0}\right)^{2}\left(1+C \alpha^{2}\right) \frac{4 \pi}{\log A(T)} .
\end{gathered}
$$

Henceforth, we take $\kappa \leq \kappa_{1}$.

To estimate $t_{0}$ in (7.2) we recall that $u=t_{0}$ on $\partial F\left(t_{0}\right)$, with $t_{0}$ as in (6.8) so that (cf. [3, p. 3])

$$
\frac{1}{t_{0}^{2}} \int_{F\left(t_{0}\right)}|D u|^{2} d x d y \geq \frac{4 \pi}{\log A\left(t_{0}\right)},
$$

that is,

$$
t_{0}^{2} \leq \frac{1}{4 \pi} \log \left(1+\kappa \alpha^{2}\right) \int_{F\left(t_{0}\right)}|D u|^{2} d x d y .
$$

By Green's theorem and the fact that $u$ is harmonic,

$$
\begin{aligned}
\int_{F\left(t_{0}\right)}|D u|^{2} d x d y & =t_{0} \int_{\partial F\left(t_{0}\right)} \frac{\partial u}{\partial n} d s \\
& =t_{0} \int_{\partial F(1)} \frac{\partial u}{\partial n} d s \\
& =t_{0} \operatorname{Cap}_{2}(\Gamma) .
\end{aligned}
$$

Thus, from (7.3) and (7.4) we have,

$$
t_{0} \leq \frac{\kappa \alpha^{2}}{4 \pi} \operatorname{Cap}_{2}(\Gamma):=M
$$

We now have two cases to examine, namely, i) $T>M$, and ii) $T \leq M$.

First we work out case i). From (7.2),

$$
\int_{F(T)}|D u|^{2} d x d y \geq \frac{4 \pi(T-M)^{2}}{\log A(T)}\left(1+C \alpha^{2}\right),
$$

We now use the usual isoperimetric inequality for $T<t<1$ as was done in (5.10) to obtain

$$
1-T \leq\left(\int_{F(1) \backslash F(T)}|D u|^{2} d x d y\right)^{1 / 2}\left(\frac{1}{4 \pi} \log \frac{4}{A(T)}\right)^{1 / 2} .
$$


This together with (7.6) and Hölder's inequality gives

$$
\begin{aligned}
4 \pi(1-M)^{2} \leq & \left(\int_{F(1)}|D u|^{2} d x d y\right) \\
& \cdot\left(\log \frac{4}{A(T)}+\frac{1}{1+C \alpha^{2}} \log A(T)\right) \\
= & \left(\int_{F(1)}|D u|^{2} d x d y\right) \\
& \cdot\left(\log 4-\frac{C \alpha^{2}}{1+C \alpha^{2}} \log A(T)\right) \\
\leq & \left(\int_{F(1)}|D u|^{2} d x d y\right) \\
& \cdot\left(1-\frac{C \alpha^{2}}{1+C \alpha^{2}} \frac{\log A(T)}{\log 4}\right) \log 4 .
\end{aligned}
$$

Now set $G=\operatorname{Cap}_{2}(\Gamma) / \operatorname{Cap}_{2}\left(\Gamma^{*}\right)$. Then $G \geq 1$. Recalling that $\operatorname{Cap}_{2}\left(\Gamma^{*}\right)$ $=4 \pi / \log 4,(7.7),(2.12)$, and $\eta=0.01$ yield

$$
(1-M)^{2} \leq G\left(1-\frac{C \alpha^{2}}{2} \frac{\log 1.01}{\log 4}\right)
$$

This together with (7.5) gives

$$
1-\frac{\kappa \alpha^{2} G}{\log 4} \leq \sqrt{G\left(1-C_{1} \alpha^{2}\right)} \leq G\left(1-C_{2} \alpha^{2}\right)
$$

Thus,

$$
G \geq \frac{1}{1-C_{2} \alpha^{2}+\kappa \alpha^{2} / \log 4} .
$$

For sufficiently small $\kappa$ we then have

$$
\mathrm{Cap}_{2}(\Gamma) \geq\left(1+C_{3} \alpha^{2}\right) \operatorname{Cap}_{2}\left(\Gamma^{*}\right)
$$

We now examine case ii), i.e. $T \leq M$. Observe that

$$
\int_{F(1)}|D u|^{2} d x d y=\frac{1}{T} \int_{F(T)}|D u|^{2} d x d y .
$$


Now, from (7.5) we deduce that

$$
T \leq \frac{\kappa \alpha^{2}}{4 \pi} \frac{1}{T} \int_{F(T)}|D u|^{2} d x d y,
$$

which in turn implies,

$$
T \leq \alpha \sqrt{\frac{\kappa}{4 \pi}}\left(\int_{F(T)}|D u|^{2} d x d y\right)^{1 / 2}
$$

By employing a procedure, similar to the one used in deriving (5.10), we again write

(7.10) $1-T \leq\left(\int_{F(1) \backslash F(T)}|D u|^{2} d x d y\right)^{1 / 2}\left(\frac{1}{4 \pi} \log \left(\frac{4}{A(T)}\right)\right)^{1 / 2}$.

Adding (7.9) and (7.10), using (2.10) and $\eta=0.01$, and applying Hölder's inequality we have

$$
\begin{aligned}
1 \leq & \left(\int_{F(1)}|D u|^{2} d x d y\right)^{1 / 2} \\
& \cdot\left(\frac{1}{4 \pi} \log \left(\frac{4}{A(T)}\right)+\frac{\kappa \alpha^{2}}{4 \pi}\right)^{1 / 2} \\
= & \left(\int_{F(1)}|D u|^{2} d x d y\right)^{1 / 2} \\
& \cdot\left(\frac{\log 4}{4 \pi}+\frac{\kappa \alpha^{2}}{4 \pi}-\frac{\log A(T)}{4 \pi}\right)^{1 / 2} \\
\leq & \left(\int_{F(1)}|D u|^{2} d x d y\right)^{1 / 2} \\
& \cdot\left(1+\frac{\kappa \alpha^{2}}{\log 4}-\frac{\log 1.01}{\log 4}\right)^{1 / 2}\left(\frac{\log 4}{4 \pi}\right)^{1 / 2} .
\end{aligned}
$$

For sufficiently small $\kappa,(7.11)$ then yields

$$
\frac{4 \pi}{\log 4} \leq\left(1-\frac{\log 1.01}{2 \log 4}\right)\left(\int_{F(1)}|D u|^{2} d x d y\right)
$$


which implies (1.5) trivially, that is, with no dependence on $\alpha$. Thus we have shown that (1.5) holds when $T>M$ and $T \leq M$, so the proof of (1.5) is complete for $p=2$.

\section{Remarks on Case 2 for $p \neq 2$.}

The procedure for obtaining the analogue of Lemma 4.1 will now follow for general $p$, with different constants, much as was done in Section 4. Inequality (4.3) becomes

$$
\begin{aligned}
I & =\int_{F_{1} \backslash \Omega_{1}}\left(v_{r}^{2}+\frac{1}{r^{2}} v_{\theta}^{2}\right)^{p / 2} r d r d \theta \\
& \geq \int_{-\pi}^{\pi}\left(\inf \int_{J(\theta) \cap\left\{F_{1} \backslash \Omega_{1}\right\}}\left|f_{r}\right|^{p} r d r\right) d \theta,
\end{aligned}
$$

where $f=f(r, \theta)$ is absolutely continuous and $f=1$ on $J(\theta) \cap \partial F_{1}$ and $f=0$ on $J(\theta) \cap \partial \Omega_{1}$. We then use the solution to the one variable Euler equation $\left(r\left|z^{\prime}\right|^{p-2} z^{\prime}\right)^{\prime}=0$ and (4.4) becomes

$$
I \geq|d|^{p-1}\left(\int_{-\pi}^{\pi} \frac{d \theta}{\left|t(\theta)^{d}-\xi(\theta)^{d}\right|^{p-1}}+\int_{E} \frac{d \theta}{\left|\hat{t}(\theta)^{d}-s(\theta)^{d}\right|^{p-1}}\right)
$$

where $d=(p-2) /(p-1)$. This follows from the observation that for $d \neq 0$ and $\xi(\theta) \leq \hat{s}(\theta) \leq t(\theta)$,

$$
\left|t(\theta)^{d}-\xi(\theta)^{d}\right|^{p-1} \geq\left|t(\theta)^{d}-\hat{s}(\theta)^{d}\right|^{p-1} .
$$

Our objective is to prove the analogue

$$
I \geq|d|^{p-1} \frac{2 \pi}{\left|R^{d}-\rho^{d}\right|^{p-1}}\left(1+K_{1} \beta^{2}-K_{2} \varepsilon^{2}-K_{3} \varepsilon \beta\right)
$$

of Lemma 4.1, where the constants $K_{1}, K_{2}$, and $K_{3}$ now depend only on $p$ for small $\varepsilon$. We first consider the case $p>2$. We write

$$
\begin{aligned}
\left(\frac{1}{t^{d}-\xi^{d}}\right)^{p-1}= & \left(\frac{1}{R^{d}-\rho^{d}}\right)^{p-1} \\
& \cdot\left(1-\frac{\left(R^{d}-t^{d}\right)-\left(\rho^{d}-\xi^{d}\right)}{\left(R^{d}-\rho^{d}\right)}\right)^{1-p}
\end{aligned}
$$


Now the condition (2.9) and (6.29) already imply that $t / R$ and $\xi / \rho$ are close to 1 ; certainly

$$
\frac{1}{2}<\frac{\xi}{\rho}, \quad \frac{t}{R}<2
$$

In addition, by $(2.9),(6.19),(6.20),(6.29 . v))-(6.29 . i x-x))$, we also have

$$
0<\frac{t^{d}-\xi^{d}}{R^{d}-\rho^{d}} \leq \sigma
$$

for some constant $\sigma=\sigma_{p}>0$, which depends only on $p$.

Let $h(x)=(1-x)^{1-p}$. Then, $h(0)=1, h^{\prime}(x)=(p-1)(1-x)^{-p}$, and $h^{\prime \prime}(x)=p(p-1)(1-x)^{-p-1}$ which is positive and increasing for $-\infty<x<1$. Using these on the interval $[1-\sigma, 1)$, we find that

$$
h(x) \geq 1+(p-1) x+h^{\prime \prime}(1-\sigma) \frac{x^{2}}{2}, \quad 1-\sigma \leq x<1 .
$$

Combining (8.4), (8.6) and (8.7), we may then write

$$
\begin{aligned}
&\left(\frac{1}{t^{d}-\xi^{d}}\right)^{p-1} \geq\left(\frac{1}{R^{d}-\rho^{d}}\right)^{p-1} \\
& \cdot(1+(p-1)( \\
&(8.8) \quad\left(\frac{R^{d}-t^{d}+\xi^{d}-\rho^{d}}{R^{d}-\rho^{d}}\right) \\
&\left.\left.+\frac{p}{2} \sigma^{-p-1}\left(\frac{R^{d}-t^{d}+\xi^{d}-\rho^{d}}{R^{d}-\rho^{d}}\right)\right)\right) .
\end{aligned}
$$

In (8.8), we shall use the following four expansions with (8.5). First we have

$$
\begin{aligned}
\int_{-\pi}^{\pi} \frac{R^{d}-t^{d}}{R^{d}-\rho^{d}} d \theta & =\frac{R^{d}}{R^{d}-\rho^{d}} \int_{-\pi}^{\pi}\left(1-\left(\left(\frac{t}{R}\right)^{2}\right)^{d / 2}\right) d \theta \\
& \geq \frac{d R^{d}}{2\left(R^{d}-\rho^{d}\right)} \int_{-\pi}^{\pi} \frac{R^{2}-t^{2}}{R^{2}} d \theta \geq 0
\end{aligned}
$$

The fact that the right hand side is nonnegative follows from (3.12). 
Also,

$$
\begin{aligned}
\int_{-\pi}^{\pi} \frac{\xi^{d}-\rho^{d}}{R^{d}-\rho^{d}} d \theta= & \frac{\rho^{d}}{R^{d}-\rho^{d}} \\
& \cdot \int_{-\pi}^{\pi}\left(\left(\left(\frac{\xi}{\rho}\right)^{2}\right)^{d / 2}-1\right) d \theta \\
\geq & \frac{d \rho^{d}}{2\left(R^{d}-\rho^{d}\right)} \int_{-\pi}^{\pi} \frac{\xi^{2}-\rho^{2}}{\rho^{2}} d \theta \\
& -2^{2-d} d\left(1-\frac{d}{2}\right)\left(\frac{\rho^{d}}{R^{d}-\rho^{d}}\right) \\
& \cdot \int_{-\pi}^{\pi}\left(\frac{\xi^{2}-\rho^{2}}{\rho^{2}}\right)^{2} d \theta,
\end{aligned}
$$

and

$$
\begin{aligned}
\int_{-\pi}^{\pi}\left(\frac{\xi^{d}-\rho^{d}}{R^{d}-\rho^{d}}\right)^{2} d \theta= & \left(\frac{\rho^{d}}{R^{d}-\rho^{d}}\right)^{2} \\
& \cdot \int_{-\pi}^{\pi}\left(\left(\left(\frac{\xi}{\rho}\right)^{2}\right)^{d / 2}-1\right)^{2} d \theta \\
\geq & d^{2} 4^{d-3}\left(\frac{\rho^{d}}{R^{d}-\rho^{d}}\right)^{2} \int_{-\pi}^{\pi}\left(\frac{\xi^{2}-\rho^{2}}{\rho^{2}}\right)^{2} d \theta .
\end{aligned}
$$

Similarly,

$$
\begin{aligned}
\int_{-\pi}^{\pi}\left(\frac{R^{d}-t^{d}}{R^{d}-\rho^{d}}\right)^{2} d \theta \geq & d^{2} 4^{d-3} \frac{\rho^{d} R^{d}}{\left(R^{d}-\rho^{d}\right)^{2}} \\
& \cdot \int_{-\pi}^{\pi}\left(\frac{R^{2}-t^{2}}{R^{2}}\right)^{2} d \theta .
\end{aligned}
$$

Using (8.8) in (8.2) we obtain

$$
\begin{aligned}
I \geq & \frac{d^{p-1}}{\left(R^{d}-\rho^{d}\right)^{p-1}} \\
& \cdot\left(2 \pi+(p-1)\left(\int _ { - \pi } ^ { \pi } \left(\frac{\xi^{d}-\rho^{d}}{R^{d}-\rho^{d}}+\frac{R^{d}-t^{d}}{R^{d}-\rho^{d}}\right.\right.\right. \\
& \left.\left.\left.\quad+\frac{p}{2} \sigma^{-p-1}\left(\frac{\xi^{d}-\rho^{d}}{R^{d}-\rho^{d}}+\frac{R^{d}-t^{d}}{R^{d}-\rho^{d}}\right)^{2}\right) d \theta\right)\right)
\end{aligned}
$$




$$
\begin{aligned}
& \geq \frac{d^{p-1}}{\left(R^{d}-\rho^{d}\right)^{p-1}} \\
& \cdot\left(2 \pi+(p-1)\left(\int _ { - \pi } ^ { \pi } \left(\frac{\xi^{d}-\rho^{d}}{R^{d}-\rho^{d}}+\frac{R^{d}-t^{d}}{R^{d}-\rho^{d}}\right.\right.\right. \\
& +\frac{p}{2} \sigma^{-p-1}\left(\left(\frac{\xi^{d}-\rho^{d}}{R^{d}-\rho^{d}}\right)^{2}+\left(\frac{R^{d}-t^{d}}{R^{d}-\rho^{d}}\right)^{2}\right. \\
& \left.\left.\left.\left.-2\left|\frac{\xi^{d}-\rho^{d}}{R^{d}-\rho^{d}}\right|\left|\frac{R^{d}-t^{d}}{R^{d}-\rho^{d}}\right|\right)\right) d \theta\right)\right) .
\end{aligned}
$$

We now use the inequalities in (8.9)-(8.12) to estimate $I$. It follows that

$$
I \geq \frac{d^{p-1}}{\left(R^{d}-\rho^{d}\right)^{p-1}}\left(2 \pi+T_{1}+T_{2}+T_{3}+T_{4}\right),
$$

where,

$$
\begin{gathered}
T_{1}=\frac{(p-1) d}{2} \frac{\rho^{d}}{R^{d}-\rho^{d}} \int_{-\pi}^{\pi}\left(\frac{R^{2}-t^{2}}{R^{2}}+\frac{\xi^{2}-\rho^{2}}{\rho^{2}}\right) d \theta, \\
T_{2}=(p-1)\left(\frac{p}{2} \sigma^{-p-1} d^{2} 4^{d-3}\left(\frac{\rho^{d}}{R^{d}-\rho^{d}}\right)-2^{2-d} d\left(1-\frac{d}{2}\right)\right) \\
\cdot \frac{\rho^{d}}{R^{d}-\rho^{d}} \int_{-\pi}^{\pi}\left(\frac{\xi^{2}-\rho^{2}}{\rho^{2}}\right)^{2} d \theta, \\
T_{3}=(p-1) \frac{p}{2} \sigma^{-p-1} d^{2} 4^{d-3} \frac{\rho^{d} R^{d}}{\left(R^{d}-\rho^{d}\right)^{2}} \int_{-\pi}^{\pi}\left(\frac{R^{2}-t^{2}}{R^{2}}\right)^{2} d \theta,
\end{gathered}
$$

and

$$
\begin{aligned}
T_{4}=-p(p-1) \sigma^{-p-1} \frac{\rho^{d} R^{d}}{\left(R^{d}-\rho^{d}\right)^{2}} \int_{-\pi}^{\pi} \mid & \left|\left(\left(\frac{\xi}{\rho}\right)^{2}\right)^{d / 2}-1\right| \\
\cdot & \left|\left(\left(\frac{t}{R}\right)^{2}\right)^{d / 2}-1\right| d \theta .
\end{aligned}
$$

Now, for some $C_{1}>0, T_{1} \geq-C_{1} A(N) / \rho^{2}$ by (3.12) and (4.10), nd $T_{3} \geq 0$. We may estimate $T_{4}$ by using, (6.29.vi)), (3.11), and (8.5) to obtain

$$
\left|T_{4}\right| \leq 200 p(p-1) \sigma^{-p-1} \frac{R^{d} \rho^{d}}{\left(R^{d}-\rho^{d}\right)^{2}} \varepsilon\left(\beta+\frac{A(N)}{\pi \rho^{2}}\right) .
$$


It is at this stage that we constrain our parameter $\eta$ for each $p \neq 2$. We now assume that $\eta$ is sufficiently small so that

(8.14) $T_{2} \geq \frac{p(p-1)}{4} d^{2} 4^{d-3} \sigma^{-p-1}\left(\frac{\rho^{d}}{R^{d}-\rho^{d}}\right)^{2} \int_{-\pi}^{\pi}\left(\frac{\xi^{2}-\rho^{2}}{\rho^{2}}\right)^{2} d \theta$.

This is possible due to $(6.29 . \mathrm{x})$ ). Using these estimates in (8.13) along with (4.15) we then obtain

$$
\begin{aligned}
I \geq \frac{d^{p-1}}{\left(R^{d}-\rho^{d}\right)^{p-1}} & \left(2 \pi+C_{3}\left(\beta-\frac{A(N)}{\pi \rho^{2}}\right)^{2}\right. \\
& \left.-C_{2} \beta \varepsilon-C_{1} \frac{A(N)}{\rho^{2}}\right),
\end{aligned}
$$

Finally, we need an estimate for $A(N)$. We first make a preliminary estimate using (8.4), (8.8), (8.9), and ignoring the second order term in (8.8). Observe that from $(8.5),\left|\left(\xi^{2}-\rho^{2}\right) / \rho^{2}\right| \leq 4$. Using this and (3.11) in (8.10), (8.8) yields

$$
\int_{-\pi}^{\pi} \frac{d \theta}{\left|t(\theta)^{d}-\xi(\theta)^{d}\right|^{p-1}} \geq \frac{2 \pi}{\left(R^{d}-\rho^{d}\right)^{p-1}}\left(1-C_{4} \beta\right) .
$$

If

$$
\begin{array}{r}
\int_{-\pi}^{\pi} \frac{d \theta}{\left|t(\theta)^{d}-\xi(\theta)^{d}\right|^{p-1}}+\int_{E} \frac{d \theta}{\left|\hat{t}(\theta)^{d}-s(\theta)^{d}\right|^{p-1}} \\
\geq \frac{2 \pi}{\left(R^{d}-\rho^{d}\right)^{p-1}}\left(1+C_{4} \beta\right)
\end{array}
$$

then (8.3) follows trivially. Otherwise, from (8.16) we have

$$
\int_{E} \frac{d \theta}{\left|\hat{t}(\theta)^{d}-s(\theta)^{d}\right|^{p-1}} \leq \frac{4 \pi}{\left(R^{d}-\rho^{d}\right)^{p-1}} C_{4} \beta .
$$

Using (6.29.vii)) to estimate $A(N)$ as in Section 4, we then obtain

$$
A(N) \leq C_{5} \varepsilon^{p} \beta R_{o}^{2}
$$

Using (8.17) in (8.15) and fixing $\eta$ so that (8.14) holds, we then obtain (8.3) with constants depending only on $p$.

A similar analysis can be carried out for $1<p<2$. 
Finally, we give the analogue of Section 4 for $p \neq 2$. Now,

$$
\begin{aligned}
\frac{2 \pi|d|^{p-1}}{\left|R^{d}-\rho^{d}\right|^{p-1}} & =\left(\int_{A\left(\Omega_{1}\right)}^{A\left(F_{1}\right)} \phi(t) d t\right)^{1-p} \\
& \geq\left(\int_{\left(1-\kappa \alpha^{2}\right)}^{A(T)} \phi(t) d t\right)^{1-p} \\
& \geq\left(\int_{1}^{A(T)} \phi(t) d t\right)^{1-p}\left(1-C_{6} \kappa \alpha^{2}\right) .
\end{aligned}
$$

By (8.3) and (8.18), there exist constants $C_{7}$ and $\kappa_{1}$ such that for $0<\kappa \leq \kappa_{1}$, we have

$$
\begin{aligned}
\int_{F(T)}|D u|^{p} d x d y \geq & \int_{F_{1} \backslash \Omega_{1}}|D u|^{p} d x d y \\
\geq & \frac{2 \pi|d|^{p-1}}{\left|R^{d}-\rho^{d}\right|^{p-1}} \\
& \cdot\left(T-t_{0}\right)^{p}\left(1+K_{1} \beta^{2}-K_{2} \varepsilon^{2}-K_{3} \varepsilon \beta\right) \\
\geq & \left(T-t_{0}\right)^{p}\left(1+C_{7} \alpha^{2}\right) \\
& \cdot\left(\int_{1}^{A(T)} \phi(t) d t\right)^{1-p} .
\end{aligned}
$$

To estimate $t_{0}$ in (8.19), we recall that $u=t_{0}$ on $\partial F\left(t_{0}\right)$ with $t_{0}$ as in (6.8), so that

$$
\frac{1}{t_{0}^{p}} \int_{F\left(t_{0}\right)}|D u|^{p} d x d y \geq\left(\int_{1}^{A\left(t_{0}\right)} \phi(t) d t\right)^{1-p}
$$

Hence,

$$
\begin{aligned}
t_{0}^{p} & \leq\left(\int_{F\left(t_{0}\right)}|D u|^{p} d x d y\right)\left(\int_{1}^{1+\kappa \alpha^{2}} \phi(t) d t\right)^{p-1} \\
& \leq C_{8}\left(\kappa \alpha^{2}\right)^{p-1} \int_{F\left(t_{0}\right)}|D u|^{p} d x d y .
\end{aligned}
$$

By Green's theorem,

$$
\int_{F\left(t_{0}\right)}|D u|^{p} d x d y=t_{0} \int_{\partial F\left(t_{0}\right)}|D u|^{p-2} \frac{\partial u}{\partial n} d s=t_{0} \operatorname{Cap}_{p}(\Gamma) .
$$


By (8.20) and (8.21),

$$
t_{0} \leq C_{9} \kappa \alpha^{2} \operatorname{Cap}_{p}(\Gamma)^{1 /(p-1)}:=M, \quad C_{9}=C_{8}^{1 /(p-1)}
$$

As in Section 7, we distinguish two possibilities, namely, i) $T>M$, and ii) $T \leq M$. Let us first assume that i) holds. Thus for $0<\kappa \leq \kappa_{1}$, (8.19) yields

$$
\int_{F(T)}|D u|^{p} \geq(T-M)^{p}\left(1+C_{7} \alpha^{2}\right)\left(\int_{1}^{A(T)} \phi(t) d t\right)^{1-p}
$$

We may now use the usual isoperimetric inequality over the interval $(T, 1)$ to obtain

$$
1-T \leq\left(\int_{F(1) \backslash F(T)}|D u|^{p}\right)^{1 / p}\left(\int_{A(T)}^{4} \phi(t) d t\right)^{(p-1) / p} .
$$

This together with (8.23) and Hölder's inequality gives us

$$
\begin{aligned}
(1-M)^{p} \leq & \left(\int_{F(1)}|D u|^{p} d x d y\right) \\
& \cdot\left(\left(\frac{1}{1+C_{7} \alpha^{2}}\right)^{1 /(p-1)} \int_{1}^{A(T)} \phi(t) d t+\int_{A(T)}^{4} \phi(t) d t\right)^{p-1} \\
= & {\left[1+\left(\left(\frac{1}{1+C_{7} \alpha^{2}}\right)^{1 /(p-1)}-1\right) \frac{\int_{1}^{A(T)} \phi(t) d t}{\int_{1}^{4} \phi(t) d t}\right]^{p-1} } \\
& \cdot\left(\int_{F(1)}|D u|^{p} d x d y\right)\left(\int_{1}^{4} \phi(t) d t\right)^{p-1} \cdot
\end{aligned}
$$

Set $Z$ to be the square bracket term on the right hand side of (8.24), and take $S=\operatorname{Cap}_{p}(\Gamma) / \operatorname{Cap}_{p}\left(\Gamma^{*}\right)$. Then $S \geq 1$, and (8.24) says that $(1-M) \leq S^{1 / p} Z^{1 / p}$, or by $(8.22)$,

$$
1-C_{9} \kappa \alpha^{2} S^{1 /(p-1)} \operatorname{Cap}_{p}\left(\Gamma^{*}\right)^{1 /(p-1)} \leq S^{1 / p} Z^{1 / p} .
$$


Since $S^{1 /(p-1)} \geq S^{1 / p}$, it follows that

$$
S^{1 /(p-1)} \geq \frac{1}{Z^{1 / p}+C_{9} \kappa \alpha^{2} \operatorname{Cap}_{p}\left(\Gamma^{*}\right)^{1 /(p-1)}}
$$

This in turn implies,

$$
\operatorname{Cap}_{p}(\Gamma) \geq\left(\frac{1}{Z^{1 / p}+C_{9} \kappa \alpha^{2} \operatorname{Cap}_{p}\left(\Gamma^{*}\right)^{1 /(p-1)}}\right)^{p-1} \operatorname{Cap}_{p}\left(\Gamma^{*}\right)
$$

Since it is easy to see that $Z \leq 1-C_{10} \alpha^{2}$, the result then follows from (8.25) for sufficiently small $\kappa$.

We next consider case ii), i.e., $T \leq M$. Now,

$$
\int_{F(1)}|D u|^{p} d x d y=\frac{1}{T} \int_{F(T)}|D u|^{p} d x d y
$$

so that by $(8.22)$,

$$
T \leq C_{9} \kappa \alpha^{2}\left(\frac{1}{T} \int_{F(T)}|D u|^{p} d x d y\right)^{1 /(p-1)} .
$$

Hence,

$$
T \leq\left(C_{9} \kappa \alpha^{2}\right)^{(p-1) / p}\left(\int_{F(T)}|D u|^{p} d x d y\right)^{1 / p} .
$$

We employ the usual isoperimetric inequality and the coarea formula over the interval $(T, 1)$ (see Section 5 ) to obtain

$$
1-T \leq\left(\int_{F(1) \backslash F(T)}|D u|^{p} d x d y\right)^{1 / p}\left(\int_{A(T)}^{1} \phi(t) d t\right)^{(p-1) / p} .
$$

This together with (8.26), (2.12), and Hölder's inequality results in

$$
1 \leq\left(\int_{F(1)}|D u|^{p} d x d y\right)\left(C_{9} \kappa \alpha^{2}+\int_{A(T)}^{1} \phi(t) d t\right)^{p-1}
$$




$$
\begin{aligned}
\leq & \left(\int_{F(1)}|D u|^{p} d x d y\right)\left(1+\frac{C_{9} \kappa \alpha^{2}}{\int_{1}^{4} \phi(t) d t}-\frac{\int_{1}^{A(T)} \phi(t) d t}{\int_{1}^{4} \phi(t) d t}\right)^{p-1} \\
& \cdot\left(\int_{1}^{4} \phi(t) d t\right)^{p-1} \\
\leq & \left(\int_{F(1)}|D u|^{p} d x d y\right)\left(1+\frac{C_{9} \kappa \alpha^{2}-\int_{1}^{1+\eta} \phi(t) d t}{\int_{1}^{4} \phi(t) d t}\right)^{p-1} \\
& \cdot\left(\int_{1}^{4} \phi(t) d t\right)^{p-1},
\end{aligned}
$$

which again gives the result for $\kappa$ sufficiently small. Thus, the proof of Theorem 1 is complete for $p \neq 2$.

\section{Sharpness of the exponent 2 .}

In this section we show that the condenser with elliptical inner set of small eccentricity gives the proper order of magnitude for capacity to show that the exponent 2 is sharp. Although there is no reason to believe that this case gives the sharp constant $K_{p}$ in Theorem 1 , it is convenient from the standpoint of calculations. On the other hand, there is some delicacy in choosing the inner set. For example, putting a small bump or a circle would result in an exponent of 1 instead of 2 on $\alpha$.

Let $\varepsilon$ be a small positive number. For each $\varepsilon$, let $E_{\varepsilon}$ denote the closed domain bounded by the ellipse $x=r_{0}(1+\varepsilon)^{1 / 2} \cos \theta, y=r_{0} \sin \theta$, where $r_{0}=1 /\left(\sqrt{\pi}(1+\varepsilon)^{1 / 4}\right)$. Then $A\left(E_{\varepsilon}\right)=1$. Let $\Gamma_{\varepsilon}$ denote the condenser $\Gamma\left(E_{\varepsilon}, \mathbb{R}^{2} \backslash B(0,2 / \sqrt{\pi})\right)$. From $[6$, p. 88-89] we have that $\alpha=$ $\alpha\left(E_{\varepsilon}\right)=\varepsilon / 2 \pi+O\left(\varepsilon^{2}\right)$, as $\varepsilon \rightarrow 0$. In order to prove our claim, we note from (1.4) and (1.5) that it is sufficient to exhibit a function $u$, belonging to the class of admissible functions for (1.4), with the property that

$$
\iint_{\mathbb{R}^{2}}|\nabla u|^{p} d x d y=\operatorname{Cap}_{p}\left(\Gamma^{*}\right)+O\left(\varepsilon^{2}\right) \quad \text { as } \varepsilon \rightarrow 0,
$$

where $\Gamma^{*}$ is as in Theorem 1. This will then imply that

$$
\operatorname{Cap}_{p}\left(\Gamma_{\varepsilon}\right)=\operatorname{Cap}_{p}\left(\Gamma^{*}\right)+O\left(\varepsilon^{2}\right) \quad \text { as } \varepsilon \rightarrow 0 .
$$


Theorem 2. Let $\varepsilon>0$, be small, $\Gamma_{\varepsilon}$ be the condenser whose inner set is $E_{\varepsilon}$ and outer set is $\mathbb{R}^{2} \backslash B(0,2 / \sqrt{\pi})$. Then for each fixed $p>1$, there is a function $u=u_{\varepsilon, p}$ with $u=0$ on $E_{\varepsilon}$ and $u=1$ on $\mathbb{R}^{2} \backslash B(0,2 / \sqrt{\pi})$, such that

$$
\iint_{\mathbb{R}^{2}}|\nabla u|^{p} d x d y=\operatorname{Cap}_{p}\left(\Gamma^{*}\right)+O\left(\varepsilon^{2}\right) \quad \text { as } \varepsilon \rightarrow 0 .
$$

Proof. We shall present details for $p \neq 2$; the case $p=2$ is similar. Set $R=2 / \sqrt{\pi}$ and $\rho=1 / \sqrt{\pi}$. Then $r_{0}=\rho /(1+\varepsilon)^{1 / 4}$. By $(1.6)$,

$$
\operatorname{Cap}_{p}\left(\Gamma^{*}\right)=\frac{2 \pi|d|^{p-1}}{\left|R^{d}-\rho^{d}\right|^{p-1}}
$$

where $d=(p-2) /(p-1)$.

Let $r, \theta$ be the polar coordinates, and define $u(r, \theta)=u_{\varepsilon, p}(r, \theta)$ as

$$
u(r, \theta)=1-\frac{R^{d}-r^{d}}{R^{d}-r_{0}^{d}\left(1+\varepsilon \cos ^{2} \theta\right)^{d / 2}},
$$

in $B(0,2 / \sqrt{\pi}) \backslash E_{\varepsilon}, u=0$ on $E_{\varepsilon}$, and $u=1$ on $\mathbb{R}^{2} \backslash B(0,2 / \sqrt{\pi})$. Then $u$ is absolutely continuous, and in $B(0,2 / \sqrt{\pi}) \backslash E_{\varepsilon}$,

$$
|\nabla u|=\frac{|d| r^{d-1}}{\left|R^{d}-r_{0}^{d}\left(1+\varepsilon \cos ^{2} \theta\right)^{d / 2}\right|}+O\left(\varepsilon^{2}\right) \quad \text { as } \varepsilon \rightarrow 0 .
$$

Then, by (9.5),

$$
\begin{aligned}
\iint_{\mathbb{R}^{2}} \mid & \left.\nabla u\right|^{p} d x d y \\
= & |d|^{p} \int_{0}^{2 \pi} \int_{r_{0} \sqrt{1+\varepsilon \cos ^{2} \theta}}^{R} \frac{r^{p /(1-p)}}{\left|R^{d}-r_{0}^{d}\left(1+\varepsilon \cos ^{2} \theta\right)^{d / 2}\right|^{p}} r d r d \theta \\
& +O\left(\varepsilon^{2}\right) \\
= & |d|^{p-1} \int_{0}^{2 \pi} \frac{\left|R^{d}-r_{0}^{d}\left(1+\varepsilon \cos ^{2} \theta\right)^{d / 2}\right|}{\left|R^{d}-r_{0}^{d}\left(1+\varepsilon \cos ^{2} \theta\right)^{d / 2}\right|^{p}} d \theta \\
& +O\left(\varepsilon^{2}\right) \\
= & |d|^{p-1} \int_{0}^{2 \pi} \frac{1}{\left|R^{d}-r_{0}^{d}\left(1+\varepsilon \cos ^{2} \theta\right)^{d / 2}\right|^{p-1}} d \theta \\
& +O\left(\varepsilon^{2}\right),
\end{aligned}
$$


as $\varepsilon \rightarrow 0$. By the definition of $r_{0}$ and $\rho$,

$$
\begin{aligned}
\left|R^{d}-r_{0}^{d}\left(1+\varepsilon \cos ^{2} \theta\right)^{d / 2}\right| & =\left|R^{d}-\rho^{d}\left(\frac{1+\varepsilon \cos ^{2} \theta}{\sqrt{1+\varepsilon}}\right)^{d / 2}\right| \\
& =\left|R^{d}-\rho^{d}+\rho^{d}\left(1-\left(\frac{1+\varepsilon \cos ^{2} \theta}{\sqrt{1+\varepsilon}}\right)^{d / 2}\right)\right| .
\end{aligned}
$$

Set

$$
h(\varepsilon)=1-\left(\frac{1+\varepsilon \cos ^{2} \theta}{\sqrt{1+\varepsilon}}\right)^{d / 2} .
$$

Now,

$$
h(\varepsilon)=-\frac{d}{2}\left(\cos ^{2} \theta-\frac{1}{2}\right) \varepsilon+O\left(\varepsilon^{2}\right) \quad \text { as } \varepsilon \rightarrow 0 .
$$

Thus, (9.7) and (9.8) imply, as $\varepsilon \rightarrow 0$,

$$
\begin{aligned}
\left|R^{d}-r_{0}^{d}\left(1+\varepsilon \cos ^{2} \theta\right)^{d / 2}\right|^{1-p}= & \left|R^{d}-\rho^{d}\right|^{1-p}\left(1+\frac{\rho^{d} h(\varepsilon)}{\left(R^{d}-\rho^{d}\right)}\right)^{1-p} \\
= & \left|R^{d}-\rho^{d}\right|^{1-p}\left(1-\frac{(p-1) \rho^{d} h(\varepsilon)}{\left(R^{d}-\rho^{d}\right)}\right) \\
& +O\left(\varepsilon^{2}\right) \\
= & \left|R^{d}-\rho^{d}\right|^{1-p} \\
& \cdot\left(1+\frac{(p-1) d \rho^{d}\left(\cos ^{2} \theta-1 / 2\right)}{2\left(R^{d}-\rho^{d}\right)} \varepsilon\right) \\
& +O\left(\varepsilon^{2}\right) .
\end{aligned}
$$

Using (9.9) in (9.6), we have, as $\varepsilon \rightarrow 0$,

$$
\begin{aligned}
\iint_{\mathbb{R}^{2}}|\nabla u|^{p} d x d y= & \frac{|d|^{p-1}}{\left|R^{d}-\rho^{d}\right|^{p-1}} \int_{0}^{2 \pi} 1+\frac{d(p-1) \rho^{d}}{2\left(R^{d}-\rho^{d}\right)}\left(\cos ^{2} \theta-\frac{1}{2}\right) \varepsilon d \theta \\
& +O\left(\varepsilon^{2}\right)
\end{aligned}
$$

Since

$$
\int_{0}^{2 \pi}\left(\cos ^{2} \theta-\frac{1}{2}\right) d \theta=0
$$

we obtain (9.2). 


\section{Logarithmic Capacity.}

We now outline the proof of (1.3). Let $\Omega$ be a compact subset of the complex plane $\mathbb{C}$ with $\partial \Omega$ a finite union of rectifiable curves. Let $G(z)$ denote Green's function for $\hat{\mathbb{C}} \backslash \Omega$ with pole at $\infty$, extended to be 0 on $\Omega$. Then

$$
-\log \operatorname{Cap}(\Omega)=\lim _{z \rightarrow \infty}(G(z)-\log |z|)
$$

For $\lambda>0$, let $\Omega_{\lambda}=\{z: G(z) \leq \lambda\}$. Then $G(z)-\lambda$ is Green's function for the complement of $\Omega_{\lambda}$. Let $\Gamma_{\lambda}$ be the condenser $\Gamma\left(\Omega, \mathbb{C} \backslash \Omega_{\lambda}\right)$. The definition of $\operatorname{Cap}\left(\Gamma_{\lambda}\right)$ is as given in (1.4) with $p=2$. In this instance, the minimizer is harmonic and is given by $G(z) / \lambda$. For $0<t \leq \lambda$, write $F(t)=\{z: G(z)<t\}$, and $A(t)=A(F(t))$. We will assume throughout that $\lambda$ is larger than some $\lambda_{0}$ in order to ensure that $A\left(\Omega_{\lambda}\right) \geq 2 A(\Omega)=$ 2. We continue to assume that $A(\Omega)=1$. In the event that $A(\Omega) \neq 1$, all areas may be scaled by $1 / A(\Omega)$ to recover the result. We will apply the coarea formula directly to $G(z)$. We take $\eta=0.01$ in $(2.10)-(2.13)$ and begin with Case 1. Set $s_{0}=\inf \{t>0: A(t) \geq 1.01\}$ and $T_{0}=$ $\sup \{t: A(t) \leq 1.02\}$. Inserting $p=2$ and $\eta=0.01$ in Lemma 5.1, we obtain

Lemma 10.1. For $\lambda \geq \lambda_{0}$, if $T_{0}$ is such that $A\left(T_{0}\right)=1.02$, then

$$
\iint_{F\left(T_{0}\right)}|D G|^{2} d x d y=\frac{4 \pi T_{0}^{2}}{\log 1.02}\left(1+D_{1} \alpha^{2}\right),
$$

where $D_{1}$ depends only on $\kappa$.

We now proceed as in Section 5. Applying the usual isoperimetric inequality over the interval $T_{0}<t<\lambda$, we obtain

$$
\left(\lambda-T_{0}\right)^{2} \leq \frac{1}{4 \pi} \log \frac{A(\lambda)}{A\left(T_{0}\right)} \iint_{\Omega_{\lambda} \backslash F\left(T_{0}\right)}|D G|^{2} d x d y .
$$

Combining this with (10.2) via Hölder's inequality, we see that

$$
\iint_{\Omega_{\lambda}}|D G|^{2} d x d y \geq \frac{4 \pi \lambda^{2}}{\log \left(A(\lambda)(1.02)^{-D_{1} \alpha^{2} /\left(1+D_{1} \alpha^{2}\right)}\right)}
$$


Since $G(z)-\log |z|$ is harmonic at $\infty$, it follows that with $r=|z|$, $\partial G / \partial r=1 / r+o\left(1 / r^{2}\right)$ as $r \rightarrow \infty$. By Green's Theorem, we have as $r \rightarrow \infty$

$$
\begin{aligned}
\iint_{\Omega_{\lambda}}|D G|^{2} d x d y & =\lambda \int_{\partial \Omega_{\lambda}} \frac{\partial G}{\partial n} d s \\
& =\lambda \int_{|z|=r} \frac{\partial G}{\partial r} d s \\
& =\lambda 2 \pi r\left(\frac{1}{r}+o\left(\frac{1}{r^{2}}\right)\right) \rightarrow 2 \pi \lambda .
\end{aligned}
$$

It follows from (10.1) that for $z \in \partial \Omega_{\lambda},|z|=\operatorname{Cap}(\Omega) e^{\lambda}(1+o(1))$, so that

$$
A(\lambda)=\pi\left(\operatorname{Cap}(\Omega) e^{\lambda}\right)^{2}(1+o(1)) \quad \text { as } \lambda \rightarrow \infty .
$$

This with (10.3) and (10.4), gives

$$
\frac{2 \pi}{\lambda} \geq \frac{4 \pi}{\log \left(\pi\left(\operatorname{Cap}(\Omega) e^{\lambda}\right)^{2}(1+o(1))(1.02)^{-D_{1} \alpha^{2} /\left(1+D_{1} \alpha^{2}\right)}\right)}
$$

Thus,

$$
\operatorname{Cap}(\Omega) \geq(1.02)^{D_{1} \alpha^{2} /\left(2\left(1+D_{1} \alpha^{2}\right)\right)} \sqrt{\frac{1}{\pi}}
$$

The inequality in (1.3) now follows in Case 1.

We now discuss Case 2. As in Section 6, we may assume that there is a $t_{0}>0$ such that (6.8)-(6.10) hold. Let $F_{1}=F_{1}(T), \Omega_{1}=$ $F_{1}(T) \cap F\left(t_{0}\right)$ as in Section 6 and let $\Gamma_{c}$ be the condenser $\Gamma\left(\Omega_{1}, \mathbb{C} \backslash F_{1}\right)$. Since $F_{1}$ and $\Omega_{1}$ are both level sets for $G(z)$, it follows that

$$
\begin{aligned}
\operatorname{Cap}\left(\Gamma_{c}\right) & =\iint_{F_{1} \backslash \Omega_{1}}|D v|^{2} d x d y \\
& =\frac{1}{\left(T-t_{0}\right)^{2}} \iint_{F_{1} \backslash \Omega_{1}}|D G|^{2} d x d y,
\end{aligned}
$$

where

$$
v(z)=\frac{G(z)-t_{0}}{T-t_{0}} .
$$


Using Lemma 4.1 in (10.5) and choosing $0<\kappa<\kappa_{0}$ for some small $\kappa_{0}$, we may show that

$$
\iint_{F(T)}|D G|^{2} d x d y \geq \frac{2 \pi\left(T-t_{0}\right)^{2}}{\log R / \rho}\left(1+B \alpha^{2}\right),
$$

where $B$ is an absolute constant. From (6.29) x), $R / \rho$ depends only on $\eta$. As was done in Case 1, we apply the usual isoperimetric inequality on $T<t \leq \lambda$, and combine the result with (10.6) via Hölder's inequality to obtain

$$
(10.7) 4 \pi\left(\lambda-t_{0}\right)^{2} \leq \log \left(A(\lambda)(1.01)^{-B \alpha^{2} /\left(1+B \alpha^{2}\right)}\right) \iint_{\Omega_{\lambda}}|D G|^{2} d x d y
$$

To estimate $t_{0}$, observe that $F\left(t_{0}\right)$ is a level set of $G(z)$, and $G(z) / t_{0}$ is harmonic in $F\left(t_{0}\right) \backslash \Omega$. Thus,

$$
\operatorname{Cap}\left(\Gamma\left(\Omega, \mathbb{C} \backslash F\left(t_{0}\right)\right)\right)=\frac{1}{t_{0}^{2}} \iint_{F\left(t_{0}\right) \backslash \Omega}|D G|^{2} d x d y \geq \frac{4 \pi}{\log A\left(t_{0}\right)}
$$

Using the inequality (6.8) and an argument similar to that in (10.4) we have

$$
t_{0}^{2} \leq \frac{1}{4 \pi} \log \left(1+\kappa \alpha^{2}\right) \iint_{F\left(t_{0}\right)}|D G|^{2} d x d y=\frac{t_{0}}{2} \log \left(1+\kappa \alpha^{2}\right)
$$

Clearly then, $t_{0} \leq \kappa \alpha^{2}$. Using (10.4), the estimate on $A(\lambda)$ (see Case 1) and the bound on $t_{0}$, in (10.7), we have

$$
4 \pi\left(\lambda-\kappa \alpha^{2}\right)^{2} \leq 2 \pi \lambda\left(\log \left(\pi\left(\operatorname{Cap}(\Omega) e^{\lambda}\right)^{2}(1+o(1))(1.01)^{-B \alpha^{2} /\left(1+\kappa \alpha^{2}\right)}\right)\right) .
$$

Simplifying the above,

$$
\operatorname{Cap}(\Omega) \geq e^{-2 \kappa \alpha^{2}}(1.01)^{B \alpha^{2} /\left(2\left(1+B \alpha^{2}\right)\right)} \sqrt{\frac{1}{\pi}}
$$

Fixing $\kappa$ such that $0<\kappa \leq \kappa_{0}$, we obtain (1.3). 


\section{The constants $K_{p}$.}

Let $\Gamma\left(\Omega, \Omega^{\prime}\right)$ be a condenser as in Section 1 , and set $\chi=A\left(\mathbb{R}^{2} \backslash \Omega^{\prime}\right)$ $/ A(\Omega)$. Let $B(0, R)$ and $B(0, \bar{R})$ be discs such that $A(B(0, R))=A(\Omega)$ and $A(B(0, \bar{R}))=A\left(\mathbb{R}^{2} \backslash \Omega^{\prime}\right)$. Let $\Gamma^{*}=\Gamma\left(\bar{B}(0, R), \mathbb{R}^{2} \backslash B(0, \bar{R})\right)$ and set $d=(p-2) /(p-1)$. Then

$$
\mathrm{Cap}_{2}\left(\Gamma^{*}\right)=4 \pi / \log \chi
$$

and, for $p \neq 2$,

$$
\operatorname{Cap}_{p}\left(\Gamma^{*}\right)=\frac{2 \pi^{p / 2}|d|^{p-1} A(\Omega)^{(2-p) / 2}}{\left|\xi^{d / 2}-1\right|^{p-1}}=\frac{2 \pi|d|^{p-1}}{\left|\bar{R}^{d}-R^{d}\right|^{p-1}} .
$$

In this section we will discuss how the constants $K_{p}=K_{p}(\chi)$ in (1.5) behave as $\chi$ varies. Note that we have taken $\chi=4$ in Theorem 1 . Although determining the dependence on $\chi$ involves only routine modifications of the proofs, this was avoided in the text since such consideration involves carrying along additional parameters and the introduction of numerous subcases. In what follows, $\hat{K}_{p}$ represents positive constants depending only on $p$. Our methods give the following:

i) $1<p<2$,

$$
K_{p}= \begin{cases}\hat{K}_{p}(\chi-1)^{2}, & 1<\chi \leq 2, \\ \hat{K}_{p}(\text { independent of } \chi), & \chi>2,\end{cases}
$$

ii) $p=2$,

$$
K_{2}= \begin{cases}\hat{K}_{2}(\chi-1)^{2}, & 1<\chi \leq 2 \\ \hat{K}_{2} / \log \chi, & \chi>2\end{cases}
$$

iii) $p>2$,

$$
K_{p}= \begin{cases}\hat{K}_{p}(\chi-1)^{2}, & 1<\chi \leq 2 \\ \hat{K}_{p} /\left|\chi^{d / 2}-1\right|, & \chi>2\end{cases}
$$

Acknowledgements. We wish to thank the referee for many helpful suggestions. 


\section{References.}

[1] Andrievski, V., Hansen, W., Nadirashvili, N., Isoperimetric inequalities for capacities in the plane. Math. Ann. 292 (1992), 191-195.

[2] Federer, H., Geometric Measure Theory. Springer-Verlag, 1969.

[3] Federer, H. and Fleming, W., Normal and integral currents. Ann. of Math. 72 (1960), 458-520.

[4] Gehring, F., Inequalities for condensers, hyperbolic capacity, and extremal length. Michigan Math. J. 18 (1971), 1-20.

[5] Hall, R., A quantitative isoperimetric inequality in $n$ dimensional space. J. Reine angew. Math. 428 (1992), 61-76.

[6] Hall, R., Hayman, W., Weitsman, A., On capacity and asymmetry. J. d'Analyse Math. 56 (1991), 87-123.

[7] Hansen, W., Nadirashvili, N., Isoperimetric inequalities for capacities, Harmonic Analysis and Discrete Potential Theory. Editor M. Picardello, Plenum Press, 1992, 193-206.

[8] Hansen, W., Nadirashvili, N., Isoperimetric inequalities in Potential Theory. Potential Analysis 3 (1994), 1-14.

[9] Hayman, W., A problem in Fourier series arising from an isoperimetric inequality. Current Problems in Analysis and Physics (Taormina 1992), 119-125, Univ. Roma, 1993.

[10] Heinonen, J., Kilpeläinen, T., Martio, O., Nonlinear Potential Theory of Degenerate Elliptic Equations. Oxford Science Publications, 1993.

[11] Lewis, J., Capacitary functions in convex rings. Arch. Rational Mech. Anal. 66 (1977), 201-224.

[12] Lewis, J., Regularity of derivatives of solutions to certain degenerate elliptic equations. Indiana Univ. Math. J. 32 (1983), 849-858.

[13] Manfredi, J., $p$-harmonic functions in the plane. Proc. Amer. Math. Soc. 103 (1988), 473-479.

[14] Maz'ya, V., Classes of domains and imbedding theorems for function spaces. Dokl. Akad. Nauk SSR 133 (1960), 527-530.

[15] Osserman, R., Bonnesen-style isoperimetric inequalities. Amer. Math. Monthly 86 (1972), 1-29.

[16] Pólya, G., Szegö, G., Isoperimetric inequalities in Mathematical Physics. Annals of Math. Studies 27, Princeton Univ. Press, 1951. 
[17] Talenti, G., Best constant in Sobolev inequality. Ann. Mat. Pura. Appl. 110 (1976), 353-372.

Recibido: 6 de octubre de 1.994

Revisado: 15 de agosto de 1.995

Tilak Bhattacharya

Indian Statistical Institute

7, S.J.S. Sansanwal Marg

New Dehli 110016

INDIA

tlk@isid.ernet.in

and

Allen Weitsman

Department of Mathematics

Purdue University

W. Lafayette, IN 47907

U.S.A.

weits@math . purdue.edu 